\title{
Consenso latinoamericano de hipertensión en pacientes con diabetes tipo 2 y síndrome metabólico*
}

\section{Latin american consensus of hypertension in patients with type 2 diabetes and metabolic syndrome}

\author{
Patricio lópez-Jaramillo, Ramiro A. Sánchez, Margarita Díaz, \\ Leonardo Cobos, Alfonso Bryce, José Z. Parra-Carrillo, \\ Fernando Lizcano, Fernando Lanas, Isaac Sinay, IVÁn D. Sierra, \\ Ernesto Peñaherrera, Mario Bendersky, Helena Schmid, \\ Rodrigo Botero, Manuel Urina, Joffre Lara, Milton C. Foos, \\ Gustavo Márquez, Stephen Harrap, Agustín J. Ramírez, \\ Alberto ZanchetTI, on behalf of the Group of Experts of Latin \\ America
}

\section{Resumen}

El presente documento ha sido preparado por un grupo de expertos, miembros de las sociedades de cardiología, endocrinología, medicina interna, nefrología y diabetes de los países de América Latina, para que sirvan de guía a los médicos que cuidan a pacientes con diabetes, hipertensión y enfermedades concomitantes o complicaciones de ambas condiciones. Aunque el concepto de síndrome metabólico actualmente es discutido, la alta prevalencia en América Latina del conjunto de alteraciones metabólicas que lo conforman sugiere que el síndrome metabólico es una entidad nosográfica común en el contexto de la medicina latinoamericana. Por lo tanto, en el presente documento se presta especial atención a este síndrome con el fin de alertar a los médicos de una particular población de alto riesgo, en la que por lo general es subestimada y no se tratan en forma optima los factores de riego que constituyen el síndrome metabólico. Las presentes recomendaciones son el resultado de las presentaciones y los debates en los paneles de discusión durante una reunión de dos días celebrada en Bucaramanga en octubre de 2012. Todos los participantes han aprobado las conclusiones finales. Los autores reconocen que la publicación y difusión de las guías no serán suficientes para alcanzar los cambios recomendados tanto en las estrategias diagnósticas como terapéuticas, por lo que se ha programado intervenciones que permitan identificar las barreras del conocimiento, de las actitudes y de comportamiento, lo que permitirá tanto a los médicos como a los pacientes una adecuada adherencia a las recomendaciones de las guías. (Acta Med Colomb 2013; 38: 154-172).

Palabras claves: hipertensión arterial, diabetes, síndrome metabólico, Consenso Latinoamericano.

\footnotetext{
Abstract

The present document has been prepared by a group of experts, members of cardiology, endocrinology, internal medicine, nephrology and diabetes societies of Latin American Countries, to serve as a guide to physicians taking care of patients with diabetes, hypertension and comorbidities or complications of both conditions. Although the concept of metabolic syndrome is currently disputed, the higher prevalence in Latin America of that cluster of metabolic alterations has suggested that metabolic syndrome is useful nosography entity in the context of Latin American medicine. Therefore, in the pres-
}

\begin{abstract}
*Traducción española autorizada para publicarse en este idioma del artículo original publicado en inglés en el Journal of Hypertension 2013; 31: 223-238.

Dr. Patricio López-Jaramillo: Fundación Oftalmológica de Santander FOSCAL, Universidad de Santander UDES, Bucaramanga (Colombia); Dr. Ramiro A. Sánchez: Unidad de Metabolismo e Hipertensión Arterial, Hospital Universitario, Fundación Favaloro, Buenos Aires (Argentina); Dra. Margarita Díaz: Clínica Platinum, Montevideo (Uruguay); Dr. Leonardo Cobos: Colegio Panamericano del Endotelio, Santiago (Chile); Dr. Alfonso Bryce: Clínica del Golf, Lima (Perú); Dr. José Z. Parra-Carrillo: Universidad de Guadalajara, Guadalajara, (México); Dr. Fernando Lizcano: Asociación Colombiana de Endocrinología, Universidad de la Sabana, Bogotá, D.C. (Colombia); Dr. Fernando Lanas: Universidad de la Frontera, Temuco (Chile); Dr. Isaac Sinay: Instituto Cardiológico de Buenos Aires, Buenos Aires (Argentina); Dr. Iván D. Sierra: Asociación Latinoamericana de Diabetes, Bogotá, D.C. (Colombia); Dr. Ernesto Peñaherrera: Hospital Luis Vernaza, Guayaquil (Ecuador); Dr. Mario Bendersky: Universidad de Córdova, Córdova (Argentina); Dra. Helena Schmid: Universidad de Rio Grande do Sul, Porto Alegre (Brasil); Dr. Rodrigo Botero: Centro Médico, Medellín, (Colombia); Dr. Manuel Urina: Sociedad Colombiana de Cardiología, Bogotá, D.C. (Colombia); Dr. Joffre Lara: Sociedad Ecuatoriana de Aterosclerosis, Guayaquil (Ecuador); Dr. Milton C. Foos: Universidad de Sao Paulo, RibeiraoPreto (Brasil); Dr. Gustavo Márquez: Federación Diabetológica Colombiana, Corozal (Colombia); Dr. Stephen Harrap: University of Melbourne, Melbourne (Australia); Dr. Agustín J. Ramírez: Unidad de Metabolismo e Hipertensión Arterial, Hospital Universitario, Fundación Favaloro, Buenos Aires (Argentina); Dr. Alberto Zanchetti: Instituto Auxológico Italiano, Milán (Italia), en nombre del Grupo de Expertos de Latinoamérica.

Correspondencia. Dr. Patricio López-Jaramillo, MD,PhD, FACP, Clínica de Síndrome Metabólico, Prediabetes y Diabetes, Dirección de Investigaciones, Fundación Oftalmológica de Santander (FOSCAL), Facultad de Medicina, Universidad de Santander (UDES), Floridablanca, Santander, (Colombia). E-mail: jplopezj@gmail.com Recibido: 22/VII/2013 Aceptado: 29/VII/2013
\end{abstract}


ent document, particular attention is paid to this syndrome in order to alert physicians on a particular high- risk population, usually underestimated and undertreated. These recommendations results from presentation and debates by discussion panels during a 2-day conference held in Bucaramanga, in October 2012, and all the participants have approved the final conclusions. The authors acknowledge that the publication and diffusion of guidelines do not suffice to achieve the recommended changes in diagnostic or therapeutic strategies, and plan suitable interventions overcoming both physicians and patients from effectively adhering to guideline recommendations. (Acta Med Colomb 2013; 38: 154-172)).

Keywords: arterial hypertension, diabetes, Latin American consensus, metabolic syndrome

\section{Introducción}

La hipertensión arterial, la diabetes y el conjunto de signos y síntomas que conforman el denominado síndrome metabólico son altamente frecuentes en América Latina y ocurren frecuentemente como condiciones asociadas. El desarrollo de recomendaciones diagnósticas y terapéuticas preparadas a través del trabajo conjunto de expertos de diferentes áreas de la medicina es deseable, considerando las bajas tasas de control alcanzadas de éstas enfermedades en el mundo real, y de los beneficios que se pueden esperar cuando se alcanzan objetivos razonables. Los recursos para el cuidado de la salud, las prioridades para la inversión de estos recursos, el nivel socio económico de la población y las prevalencias de hipertensión, diabetes tipo 2 y otras enfermedades relacionadas, varían considerablemente en diferentes regiones del mundo, también en diferentes países de una misma región, y aun más en diferentes áreas de un mismo país. Por tanto, las recomendaciones para ser implementadas de forma útil en la práctica médica diaria deben considerar las características particulares médicas y sociales de la región o del país donde ellas deben ser aplicadas, y deben ser costo-efectivas en términos de las necesidades locales y de las posibilidades financieras y técnicas. Por estas razones, la Organización Mundial de la Salud (OMS), la Sociedad Internacional de Hipertensión (ISH) (1) y la Sociedad Europea de Hipertensión (ESH) junto con la Sociedad Europea de Cardiología (ESC) (2) han propuesto, en diferentes documentos, la necesidad de desarrollar guías regionales. Además, la aceptación y el uso de las guías regionales probablemente sean mejor aceptadas si los médicos y expertos locales están participando del desarrollo y subsecuente difusión e implementación de las guías $(3,4)$. Es por esto que este documento ha sido preparado por un grupo de expertos, miembros de Sociedades Nacionales y Latinoamericanas de Cardiología, Endocrinología, Nefrología, Medicina Interna y Diabetes, para servir como una guía para los médicos que toman cuidado de los pacientes con diabetes, hipertensión y comorbilidades, o de las complicaciones de ambas condiciones. A pesar que el concepto de "Síndrome Metabólico" es actualmente controvertido, la alta prevalencia en América Latina de ese grupo de alteraciones metabólicas determina que el síndrome metabólico sea una patología común en el contexto de la medicina latinoamericana. Por lo tanto, en el presente documento se presta especial atención a este síndrome con el fin de alertar a los médicos en una población particular de alto riesgo, generalmente subestimada y subtratada.

Estas recomendaciones son el resultado de las presentaciones y debates de los paneles de discusión durante una reunión de dos días celebrada en Bucaramanga, en octubre de 2012. Los consultores y moderadores de las sesiones plenarias fueron el Dr. Stephen Harrap y el Dr. Alberto Zanchetti, y todos los participantes aprobaron las conclusiones finales.

Los autores reconocen que la publicación y difusión de las guías no son suficientes para alcanzar los cambios recomendados en el diagnóstico o en estrategias terapéuticas,y han programado acciones apropiadas que permitan superar las barreras comportamentales tanto de los médicos como de los pacientes, lo que permitirá de manera efectiva la adhesión a las recomendaciones de las guías $(5,6)$.

Existe una gran diversidad de las características socioeconómicas en los países de América Latina, y ésto se refleja en diferencias en las tasas de morbimortalidad cardiovascular. A diferencia de lo que ha ocurrido en los Estados Unidos y en Europa Occidental, en la mayoría de los países de América Latina, la tasa de mortalidad cardiovascular ha aumentado durante las últimas décadas del siglo XX y comienzos del siglo XXI, con la excepción de Argentina y Uruguay. Pero incluso en estos últimos países, la morbilidad cardiovascular y la prevalencia de los factores de riesgo cardiovascular se han mantenido sin cambios o han aumentado, especialmente la hipertensión arterial, la obesidad, el síndrome metabólico y la diabetes $(7,8)$. De hecho, años antes que se presente el actual incremento de las enfermedades cardiovasculares, se dieron los cambios de estilo de vida en la región, especialmente de hábitos alimentarios tradicionales hacia modelos occidentales de la nutrición, lo que puede haber facilitado la expresión genética de esta enfermedades (9). Además, los patrones de morbilidad se complica aún más por el fenómeno de la migración progresiva de la población rural a las zonas urbanas, lo que incrementa la periferia urbana con una población de bajos ingresos individuales, lo que favorece la presencia de factores de riesgo emergentes como la aculturación, la violencia, el estrés y la desnutrición (7). 


\section{Prevalencia de hipertensión arterial en América Latina}

Los factores de riesgo cardiovascular se definen como características biológicas o formas de vida que aumentan la probabilidad (riesgo) de morbilidad y mortalidad cardiovascular (10). Como factores de riesgo cardiovascular, la hipertensión generalmente integra un conjunto de factores de riesgo definidos, operativamente, como el síndrome metabólico. Entre estos factores de riesgo, la hipertensión arterial es la primera causa de mortalidad a nivel mundial, y la tercera causa de incapacidad inducida por enfermedad después de la desnutrición y de las enfermedades sexuales (11).

La Tabla 1 muestra la prevalencia, conocimiento, tratamiento y control de la hipertensión arterial en América Latina. La prevalencia de la hipertensión (12-14) fue similar en Argentina (25-36\%), Uruguay (30\%), Paraguay (21-30\%) y el sur de Brasil (31-33\%). En Chile (15) se encontraron diferencias dependiendo del nivel socio-económico (bajas condiciones: $24.5 \%$, altas condiciones: $17.9 \%$ ). En México se observaron diferencias dependiendo de las zonas: $30 \%$ a

Tabla 1. Tasas de conocimiento, tratamiento y control de hipertensión arterial en América Latina.

\begin{tabular}{|c|c|c|c|c|c|c|c|c|}
\hline País & Lugar & $\begin{array}{c}\text { Año de } \\
\text { publicación }\end{array}$ & $\begin{array}{c}\text { Edad } \\
\text { (Años) }\end{array}$ & $\begin{array}{c}\text { Total } \\
\text { número }\end{array}$ & $\begin{array}{c}\text { Pacientes } \\
\text { hipertensos }(\%)\end{array}$ & $\%$ Conciencia & $\%$ Tratado & $\%$ Controlado \\
\hline \multirow[t]{9}{*}{ Argentina } & La Plata & 1988-1989 & $15-75$ & 6386 & 32.3 & $44.0(42.8-45.2)$ & $33.1(31.0-35.2)$ & $5.0(4.3-5.4)$ \\
\hline & Rauch & 1992 & $15-75$ & 1523 & 35.7 & $36.5(35.5-37.5)$ & $32.7(31.1-32.9)$ & $4.0(2.6-6.0)$ \\
\hline & Lujan & 1995 & $18-79$ & 2475 & 24.6 & $56.9(55.7-58.1)$ & $54.2(53.0-55.4)$ & $23.0(22.0-24.0)$ \\
\hline & Córdoba & 1999 & $15-85$ & 6875 & 29.9 & $54.9(52.4-57.4)$ & $43.0(40.5-45.5)$ & $13.0(11.3-14.8)$ \\
\hline & Dean Funes & 1999 & $20-70$ & 750 & 29.7 & $19.3(14.4-25.1)$ & $6.7(3.8-10.8)$ & - \\
\hline & Rosario & 1999 & $21-65$ & 2071 & 31.3 & $79.7(78.1-81.3)$ & $47.8(45.8-49.8)$ & $25.3(23.3-26.8)$ \\
\hline & Rural/Urbana & NP & $19-99$ & 10415 & 26.0 & $50.8(48.6-53.0)$ & $41.7(39.6-43.8)$ & $13.0(11.3-14.8)$ \\
\hline & Buenos Aires & 2005 & $25-64$ & 1482 & 29.0 & $64.1(59.9-68.2)$ & $41.6(37.5-45.8)$ & $18.0(14.8-21.2)$ \\
\hline & Porto Alegre & 1994 & $>18$ & 1091 & 29.7 & $39.1(33.4-44.6)$ & $13.8(10.3-18.1)$ & - \\
\hline Brasil & Sao Paulo (NE) & 2001 & $>18$ & 688 & 31.5 & $77.0(70.7-82.4)$ & $61.8(54.9-68.3)$ & $17.0(12.3-22.7)$ \\
\hline Colombia & Bogotá & 2005 & $25-64$ & 1553 & 13,5 & $68.8(62.5-75.5)$ & $55.0(48.2-61.8)$ & $30.6(25.8-35.5)$ \\
\hline \multirow[t]{4}{*}{ Chile } & Concepción & 1988 & $>14$ & 10139 & 18.6 & $65.7(63.5-67.8)$ & $30.0(27.9-32.2)$ & $7.5(6.4-8.7)$ \\
\hline & Concepción & 2004 & $>15$ & 8472 & 21.6 & $66.6(\mathrm{NR})$ & 59.9 (NR) & 30.7 (NR) \\
\hline & Valparaíso & 1999 & $25-69$ & 3120 & 11 & $44.0(42.2-45.8)$ & $22.0(20.5-23.5)$ & - \\
\hline & Santiago & 2005 & $25-69$ & 1655 & 23.8 & $61.1(55.4-64.7)$ & $43.0(38.8-47.7)$ & $20.3(16.4-24.2)$ \\
\hline Cuba & Nacional & NR & NR & 102235 & 39.7 & $70.2(\mathrm{NR})$ & - & $39.7(39.2-40.2)$ \\
\hline \multirow[t]{2}{*}{ Ecuador } & Nacional & 1999 & $>18$ & 10605 & 28.6 & $41.0(37.7-43.4)$ & $23.0(22.3-23.8)$ & $7.0(6.5-7.5)$ \\
\hline & Quito & 2005 & $25-64$ & 1638 & 8.6 & $67.6(60.2-74.9)$ & $51.8(43.9-59.8)$ & $28.0(19.9-36.1)$ \\
\hline \multirow[t]{6}{*}{ México } & Guadalajara & 1980 & $>16$ & 4031 & 21.5 & $51.3(47.9-54.7)$ & $45.6(42.3-49.1)$ & $7.6(6.0-9.6)$ \\
\hline & Aguas Calientes & 1997 & $>25$ & 6128 & 26.8 & $75.0(73.9-76.1)$ & $37.0(35.8-39.2)$ & - \\
\hline & Durango & 1998 & $>20$ & 5802 & 21.9 & $69.1(67.9-70.3)$ & - & - \\
\hline & Norte (Rural) & 2000 & $25-64$ & 815 & 6.8 & $41.0(37.5-44.5)$ & - & - \\
\hline & Nacional & 2000 & $25-64$ & 38377 & 31.3 & $43.0(42.1-43.9)$ & $20.3(17.9-22.9)$ & $4.9(3.7-6.3)$ \\
\hline & México DF & 2005 & $25-64$ & 1722 & 11.6 & $75.7(70.1-81.2)$ & $65.7(60.4-70.9)$ & $41.0(36.2-45.8)$ \\
\hline Paraguay & Nacional & 1995 & $18-74$ & 9880 & 30,4 & $11.0(10.4-11.7)$ & $5.5(5.1-6.0)$ & 0.0 \\
\hline Perú & Lima & 2005 & $25-64$ & 1652 & 12,5 & $53.1(46.5-59.6)$ & $28.8(24.0-33.5)$ & $12.0(8.4-15.7)$ \\
\hline Uruguay & Minas & NR & $>18$ & 560 & 37,3 & $78.5(72.2-83.9)$ & $47.4(40.4-54.3)$ & $16.3(11.5-22.0)$ \\
\hline \multirow[t]{3}{*}{ Venezuela } & Barquisimeto & 1994 & $>20$ & 15000 & 23,5 & $61.3(60.5-62.1)$ & $46.0(44.4-47.6)$ & $20.6(19.2-22.0)$ \\
\hline & Barquisimeto & 2000 & $>20$ & 7424 & 36,8 & $45.7(44.7-46.8)$ & $22.9(21.9-23.9)$ & $4.5(4.0-5.0)$ \\
\hline & Maracaibo & 2005 & $25-64$ & 1848 & 24,6 & $72.0(67.8-76.2)$ & $48.9(44.2-53.5)$ & $20.7(17.4-24)$ \\
\hline
\end{tabular}


nivel urbano, $11.7 \%$ a nivel rural (16). El estudio reciente (17 y Chow et al. JAMA 2013, in press), Prospectivo Urbano Rural Epidemiología (PURE) incluyó 153996 adultos (35-70 años) de 628 comunidades rurales y urbanas de tres países de ingresos altos (PIA), siete países de ingresos medios altos (PIMA), tres países de ingresos medios bajos (PIMB) y cuatro países de ingresos bajos (PIB) en diversas partes del mundo. La hipertensión se definió cuando los individuos dijeron que recibían tratamiento para la hipertensión o tenían un promedio de presión arterial (PA) mayor de 140/90mmHg en dos mediciones de PA tomadas en posición sentada en reposo utilizando un dispositivo digital automatizado. Globalmente $40.7 \%$ de los participantes tuvieron hipertensión, con el $13.3 \%$ presentando una presión arterial de al menos $160 / 100 \mathrm{mmHg}$ y $4.4 \%$ una presión arterial de al menos 180/110mmHg. De las personas con hipertensión, el 46.4\% eran conscientes de ésta condición, el $40.6 \%$ estaban en tratamiento farmacológico, pero sólo el $13.1 \%$ tenían la PA controlada $(<140 / 90 \mathrm{mmHg})$.

La prevalencia de hipertensión arterial fue similar en los PIMA (46.6\%), PIA (40.7\%) y PIMB (39.6\%), pero más baja en los PIB (32.2\%). Los porcentajes de conciencia de la enfermedad fueron PIA: 49.1\%, PIMA: $52.4 \%$, PIMB: $43.5 \%$ y PIB: $40.8 \%$ (tendencia $=\mathrm{P}<0.001$ ), el porcentaje de pacientes en tratamiento fueron $46.8,48.3,36.8$ y $31.7 \%$, respectivamente(tendencia $=\mathrm{P}<0.001$ ) y el porcentaje de pacientes que tenían la presión controlada fueron 19, 15.5, 9.9 y $12.7 \%$, respectivamente (tendencia $=\mathrm{P}<0.001$ ) lo que demuestra que estos porcentajes fueron inversamente relacionada con el nivel económico del país.

La prevalencia, el conocimiento, los pacientes tratados $\mathrm{y}$ controlados fueron mayores en las zonas urbanas que en las comunidades rurales de los PIB y en los PIMB, pero esto no ocurrió en los PIA y PIMA. En general, el $12.5 \%$ de los pacientes hipertensos tratados recibieron dos o más medicamentos para bajar la presión arterial, con una tendencia decreciente a partir de los países ricos a los países más pobres (PIA 18.1\%, PIMA 14.5\%, PIMB 14.1\%, PIB 1.6\%, $\mathrm{p}<0.0001)$. El bajo nivel de educación está fuertemente asociado con tasas más bajas de conocimiento, tratamiento y control de la presión arterial en los países de menor nivel económico, pero esto es menos evidente en los países de mayores ingresos. La prevalencia de hipertensión fue mayor en los participantes con diabetes (63\%), y a pesar de que la conciencia de la enfermedad fue de $74.4 \%$ y que el porcentaje de los diabéticos hipertensos que recibían tratamiento fue de $69.3 \%$, el porcentaje de pacientes con adecuado control fue sólo $23.3 \%$. El análisis por regiones indica que la prevalencia de hipertensión fue mayor en África (56.6\%), seguido por Malasia (46.5\%) y América del Sur (46.5\%).

Los países de América del Sur incluidos en el estudio PURE fueron Argentina, Brasil, Colombia y Chile. La Tabla 2 muestra las características de los individuos estudiados según el país. Los porcentajes de conocimiento, tratamiento y control de la hipertensión en los cuatro países de América del Sur en promedió fueron $57.0 \%, 52.8 \%$ y $18.3 \%$, respectivamente (Chow et al., JAMA 2013, in press).

De los datos analizados se puede concluir que en todo el mundo la detección y el tratamiento de la hipertensión son deficientes, y que incluso la mayoría de los pacientes que están siendo tratados tienen mal control de la PA. Estos hallazgos fueron comunes en todos los países, independiente de los niveles socio-económicos, aunque el porcentaje de tratamiento y el control fueron marcadamente peores en los PIB. Por lo tanto, deben realizarse esfuerzos sistemáticos para la implementación a nivel comunitario de programas de detección eficientes, a través de la implementación de estrategias basadas en algoritmos simples que permitan un mejor tratamiento y control de la PA, programas que son cruciales para reducir la carga de las enfermedades relacionadas con la hipertensión.

\section{Prevalencia del síndrome metabólico en América Latina}

En América Latina la prevalencia de los componentes del síndrome metabólico, como la hipertensión arterial, parece ir en aumento. Un gran cuerpo de estudios locales (18-41) ha reportado que la prevalencia de síndrome metabólico en adultos oscila entre 25 a $45 \%$, con diferencias importantes entre las zonas urbanas y rurales, pero las comparaciones son difíciles debido a las diferentes definiciones utilizadas. En pacientes con infarto agudo de miocardio o con accidente

Tabla 2. Características de los participantes de América del Sur por país.

\begin{tabular}{|c|c|c|c|c|c|c|c|c|c|}
\hline País & Número & $\begin{array}{l}\text { Reclutado } \\
\text { (Años) }\end{array}$ & $\begin{array}{c}\text { Rural } \\
{[\mathbf{n}=(\%)]}\end{array}$ & $\begin{array}{r}\text { Femenino } \\
{[\mathrm{n}=(\%)]}\end{array}$ & $\begin{array}{c}\text { Edad } \\
(A \tilde{n} o s, S D)\end{array}$ & $\begin{array}{c}\text { SBP } \\
(\mathrm{mmHg}, \mathrm{SD})\end{array}$ & $\begin{array}{c}\text { DBP } \\
(\mathrm{mmHg}, \mathrm{SD})\end{array}$ & $\begin{array}{c}\mathrm{BP} \geq 140 / \\
90 \mathrm{mmHg}[\mathrm{n}=(\%)]\end{array}$ & $\begin{array}{c}\mathrm{BP} \geq 160 / \\
100 \mathrm{mmHg}[\mathrm{n}=(\%)]\end{array}$ \\
\hline Argentina & 7483 & 2006-2009 & $3894(52.0)$ & $4603(61.5)$ & $51(10.0)$ & $135.6(21.7)$ & $28.75(12.5)$ & $3804(50.8)$ & $2455(32.6)$ \\
\hline Brasil & 5566 & 2005-2009 & $1300(23.4)$ & $3076(53.3)$ & $52(9.4)$ & $132.33(23.8)$ & $86.63(38.0)$ & $2928(52.6)$ & $2274(37.5)$ \\
\hline Chile & 3112 & 2006-2009 & $643(20.0)$ & $2135(66.5)$ & $52(9.8)$ & $130.80(22.2)$ & $82.11(20.4)$ & $1499(46.7)$ & $1058(30.7)$ \\
\hline Colombia & 7417 & 2005-2009 & $3964(53.4)$ & $4759(64.2)$ & $51(9.7)$ & $128.77(23.3)$ & $81.05(16.9)$ & $2781(37.5)$ & $1737(23.3)$ \\
\hline
\end{tabular}


cerebrovascular (27), la prevalencia fue tan alta como el 75\%, independientemente de los criterios de diagnóstico utilizados para síndrome metabólico (International Diabetes Federación, IDF, o panel de tratamiento del adulto III, ATP III).

Un reciente meta-análisis que incluyó 12 estudios transversales realizados en los países latinoamericanos (42) mostró que la prevalencia general (media ponderada) de síndrome metabólico (SM) según los criterios del ATP III fue $24.9 \%$ (rango: 18.8-43.3\%). El SM fue ligeramente más frecuente en mujeres $(25.3 \%)$ que en hombres $(23.3 \%)$ y el grupo de edad con mayor prevalencia fue el de mayores de 50 años. Los componentes más frecuentes de síndrome metabólico fueron bajas concentraciones del colesterol de lipoproteína de alta densidad (colesterol HDL; 62.9\%) y obesidad abdominal (45.8\%). Hallazgos similares se reportaron en el estudio multicéntrico CARMELA realizado en ciudades capitales de los países de América Latina (21).

\section{Prevalencia de diabetes tipo 2 en América Latina}

En la población urbana de América Latina la prevalencia de diabetes está entre 4 y 8\%, y es mayor en los países o zonas con un nivel socio-económico bajo o medio (Tabla 3). Sin embargo, los datos son escasos y el porcentaje de pacientes sin confirmación del diagnóstico es alrededor del $30-50 \%$ y puede ser mayor en las zonas rurales. El estudio CARMELA (12) realizado en siete ciudades de países de América Latina durante el año 2005 encontró que la prevalencia de diabetes tuvo casi el doble de los valores previamente reportados. La prevalencia de diabetes fue de $6 \%$ en Barquisimeto, Venezuela, $8 \%$ en Bogotá, Colombia, $6.2 \%$ en Buenos Aires, Argentina, $8.9 \%$ en México DF, México, y $7.2 \%$ en Santiago, Chile. Al igual que en otras áreas del mundo, la creciente prevalencia de diabetes en América Latina se debe, principalmente, a los cambios en los estilo de vida: menor actividad física, mayor consumo de calorías y aumento de la prevalencia de sobrepeso / obesidad, decurrentes de la acelerada urbanización.

En sujetos con diabetes la prevalencia de hipertensión arterial es 1.5-3 veces mayor que en los individuos no diabéticos con edad similar, asociación particularmente alta en países de medianos y bajos ingresos (12, 43-48).

\section{Prevalencia de sobrepeso y obesidad en América Latina}

Diferentes encuestas realizadas en América Latina (44-61) reportan una importante prevalencia de personas con sobrepeso $\left(\mathrm{IMC} 25-29.9 \mathrm{~kg} / \mathrm{m}^{2}\right.$ ) y obesidad $\left(\mathrm{IMC} \geq 30 \mathrm{~kg} / \mathrm{m}^{2}\right)$.
Tabla 3. Prevalencia de diabetes mellitus en América Latina.

\begin{tabular}{|l|c|}
\hline Ciudad & $\%$ \\
\hline Argentina & 5.0 \\
Bolivia & 5.0 \\
Brasil & 7.6 \\
Colombia & 7.3 \\
Cuba & 4.5 \\
Chile & 3.9 \\
Jamaica & 13.4 \\
México & 8.6 \\
Paraguay & 6.2 \\
Uruguay & 7.0 \\
Venezuela & 4.4 \\
\hline
\end{tabular}

En Rosario, Argentina (48), la prevalencia de sobrepeso fue de $40 \%$ y la de obesidad de $29 \%$. En la ciudad de Río de Janeiro (55), el sobrepeso estuvo presente en $40 \%$ y la obesidad en $21 \%$ de la población estudiada. En México (43, 49) la prevalencia de sobrepeso fue de $37 \%$ en las zonas rurales y $48 \%$ en México DF y la obesidad fue alrededor de 21\% (rural: 7\%, DF: 29\%). En Cuba (54), el sobrepeso y la obesidad en conjunto alcanzaron cifras de alrededor $22 \%$. En muchos estudios hubo una fuerte asociación entre obesidad e hipertensión arterial con un $40 \%$ de los individuos presentando hipertensión arterial y obesidad.

Las estimaciones de la prevalencia específica de obesidad han demostrado una gran variabilidad entre las poblaciones latinoamericanas, que van desde 9.9 hasta $35.7 \%$ (57). Las mujeres $(23,33,37,51)$ y las personas que habitan en las zonas urbanas (41) han sido identificadas como los grupos predominantemente afectados. Además, la obesidad ha sido relacionada de forma independiente con bajo estatus socioeconómico y bajos niveles educativos $(49,53)$, lo que contribuye a las desigualdades en salud observadas en la región $(59,60)$. Sin embargo, hay evidencia de una tendencia secular hacia el aumento de la prevalencia de obesidad en los países económicamente más desarrollados de América Latina (61).

$\mathrm{Al}$ igual que en los adultos, la obesidad se ha convertido también en un problema de salud en los niños de América Latina, debido al alto riesgo de persistencia de la obesidad en la edad adulta asociada al desarrollo de hipertensión arterial $(22,50,51)$. 


\section{Síndrome metabólico, diabetes e hipertensión arterial: definición, diagnóstico y evaluación clínica}

\section{Síndrome metabólico}

Como se mencionó anteriormente, el concepto de síndrome metabólico esta controvertido principalmente porque es difícil probar que el riesgo cardiovascular en las personas con síndrome metabólico es mayor que el atribuible a la suma del riesgo atribuible a cada uno de sus componentes. El síndrome metabólico es un cuadro clínico con características fácilmente detectables, y a pesar de esto todavía permanece insuficientemente diagnosticado, que tiene la ventaja que bajo un solo término agrupa un conjunto de alteraciones metabólicas muy prevalentes en América Latina. Por lo tanto, es un instrumento útil para identificar a individuos en alto riesgo de enfermedad cardiovascular (ECV) y de diabetes. Actualmente está bien aceptado que todos los componentes del síndrome metabólico están asociadas con resistencia a la insulina $(26,62,63)$.

El reciente consenso del Grupo de Trabajo en Epidemiología y Prevención de la Federación Internacional de Diabetes (FID), Instituto Nacional del Corazón, Pulmón y Sangre de los Estados Unidos, Asociación Americana del Corazón, Federación Mundial del Corazón, Sociedad Internacional de Aterosclerosis y Asociación Internacional para el Estudio de la Obesidad (62) propuso que la presencia de tres de los cinco criterios siguientes hacen el diagnóstico de síndrome metabólico:

1. Circunferencia de cintura elevada, cuya definición es específica para cada población y cada país;

2. Triglicéridos iguales o mayores a $150 \mathrm{mg} / \mathrm{dL}$, o tratamiento farmacológico para la elevación de los triglicéridos;

3. Reducción de colesterol HDL a menos de $40 \mathrm{mg} / \mathrm{dL}$ en hombres y menos de $50 \mathrm{mg} / \mathrm{dL}$ en mujeres. (El tratamiento con medicamentos para reducir el colesterol HDL tal como ácido nicotínico es un indicador alternativo);

4. Presión Arterial en el rango alto de la normalidad o hipertensión (PAS $\geq 130 \mathrm{mmHg}$ y/o PAD $\geq 85 \mathrm{mmHg}$ ) o en tratamiento farmacológico actual con antihipertensivos

5. Glucosa venosa en ayunas igual o mayor a $100 \mathrm{mg} / \mathrm{dL}$ o en tratamiento farmacológico con hipoglucemiantes.

Varios autores consideran que la obesidad central (abdominal) es el principal factor en el síndrome metabólico y debería incluirse como obligatorio en su diagnóstico. Para definir el punto de corte de obesidad abdominal en América Latina, un estudio reciente (64), el cual incluyó las capitales de varios países, recomendó valores de cintura iguales o mayores a $94 \mathrm{~cm}$ para los hombres y $88 \mathrm{~cm}$ en las mujeres. Sin embargo, un importante número de estudios independientes realizados en diferentes poblaciones de América Latina, han demostrado que los puntos de corte sugeridos por la FID (90 cm para hombres y de $80 \mathrm{~cm}$ para mujeres) son los que mejor se relacionan con la presencia de los otros componentes del síndrome metabólico (27, 28, 30, 34, 36).

A pesar de que no existen estudios de cohorte en América Latina que evalúen la relación de los puntos de corte de la circunferencia de la cintura con el desarrollo de diabetes o de enfermedades cardiovasculares es de esperarse que, como ocurre con la mayoría de los otros factores de riesgo cardiovascular, la relación sea continua y que cualquier punto de corte se basa en convenciones arbitrarias. La elección de los autores de este documento de consenso es el de usar los valores de corte propuestos por la FID $(90 \mathrm{~cm}$ para hombres y de $80 \mathrm{~cm}$ para mujeres).

Los factores de riesgo que se asocian con un mayor riesgo de síndrome metabólico se enumeran a continuación:

1. Antecedentes familiares de diabetes mellitus tipo 2

2. Diabetes mellitus gestacional

3. Macrosomía

4. Bajo peso al nacer

5. Desnutrición infantil

6. Alta mortalidad perinatal y/o presencia temprana de enfermedad cardiovascular en familiares de primer grado;

7. Hábito sedentario

8. Dieta rica en grasa animal

9. Raza

10. Bajo nivel socioeconómico

11. Historia de dislipidemia, obesidad e hipertensión

12. Hiperandrogenismo en la mujer, y

13. Acantosis nigricans.

El diagnóstico de síndrome metabólico puede ser útil para la identificación de sujetos en los cuales se puede realizar prevención primaria de diabetes mellitus, hipertensión y ECV. Se espera que un aumento en la detección mejore la conciencia tanto en los pacientes como en los médicos del riesgo cardiometabólico que corren estos individuos, y en consecuencia sea útil para reforzar la motivación para realizar los cambios necesarios en los estilos de vida y en la reducción de peso. Al momento no existen estudios que demuestren que el tratamiento del síndrome metabólico con medicamentos sea útil, sin embargo es claro que cuando la PA, los lípidos y la glucosa en plasma están por encima del umbral aceptado para definir hipertensión, dislipidemia y diabetes, el tratamiento medicamentoso antihipertensivo, antilipídico y antidiabético debe ser iniciado.

\section{Diabetes tipo 2}

Los criterios para el diagnóstico de la diabetes mellitus tipo 2, aprobadas y recomendadas por el Consenso Latinoamericano, son las siguientes:

1. Glucosa plasmática en ayunas igual o mayor a $126 \mathrm{mg} /$ $\mathrm{dL}$ en dos lecturas sucesivas. 
2. Glucosa plasmática igual o mayor a $200 \mathrm{mg} / \mathrm{dL}$ después de 120 minutos de administrados $75 \mathrm{~g}$ de glucosa en la prueba de sobrecarga oral a la glucosa.

3. Glucosa plasmática igual o mayor a $200 \mathrm{mg} / \mathrm{dL}$ en cualquier momento del día en presencia de síntomas.

Estos son los mismos criterios de la Asociación Americana de Diabetes (ADA) (65) los cuáles fueron adoptados por el Consenso, sin embargo se destacó la importancia del test de tolerancia oral a la glucosa (TTOG) como una herramienta diagnóstica más especifica. Se consideró que si bien el recién revivido termino de "prediabetes" y los menores puntos de corte para definir intolerancia a la glucosa (glucosa en ayunas alterada (GAA: $100-125 \mathrm{mg} / \mathrm{dL}$ ) y/o TTOG alterado (TTOG: $140-199 \mathrm{mg} / \mathrm{dL}$ )) pueden mejorar la detección de la diabetes $(66,67)$, la costo-efectividad de estas estrategias en términos de implementación de programas de tratamiento y prevención de las complicaciones es todavía desconocido (68), por lo cuál el Consenso prefirió recomendar la clasificación de la ADA (65).

\section{Hipertensión: clasificación y diagnóstico}

Después de considerar las clasificaciones propuestas por el Séptimo Reporte del Comité Nacional Conjunto en Prevención, Detección, Evaluación y Tratamiento de la Hipertensión Arterial (69), las guías de la ESH-ESC 2007 sobre el manejo de la hipertensión (70), la revaluación del 2009 de la Guías Europeas (71) y el anterior Consenso Latinoamericano de Hipertensión Arterial (10), se decidió, como se muestra en la Tabla 4, mantener el concepto que la hipertensión arterial se diagnostica cuando los valores de presión arterial son iguales o mayores a 140 o $90 \mathrm{mmHg}$ tomadas en el consultorio del médico o en una clínica. Por encima de este valor, la hipertensión puede ser subdividida en grados 1, 2 o 3. Esta clasificación también se aplica para la hipertensión sistólica aislada, la cual debe ser diagnosticada y tratada, especialmente en pacientes ancianos. Los pacientes ancianos mayores de 80 años deben ser diagnosticados como hipertensos cuando la PA es igual o mayor a 150/90mmHg. En pacientes de edad avanzada la PA también debe medirse en posición de pie para detectar un posible descenso ortostático excesivo.

La hipertensión arterial se clasifica como primaria, esencial o idiopática, cuando la PA es consistentemente más

Tabla 4. Clasificación de la presión arterial e hipertensión recomendado por el Consenso Latinoamericano.

\begin{tabular}{|l|c|}
\hline Presión arterial & Valor $(\mathbf{m m H g})$ \\
\hline Optima<120/80 & \\
\hline Normal & $120 / 80-129 / 84$ \\
Normal alta & $130 / 85-139 / 89$ \\
Hipertensión grado 1 & $140 / 90-159 / 99$ \\
Hipertensión grado 2 & $160 / 100-179 / 109$ \\
Hipertensión grado 3 & $\geq 180 / 110$ \\
Hipertensión sistólica aislada & $\geq 140 /<90$ \\
\hline
\end{tabular}

alta que lo normal, sin causa conocida, la cual representa más de $90 \%$ de todos los casos. Se define como hipertensión secundaria cuando la PA se eleva como resultado de una causa subyacente, identificable y con frecuencia corregible (el restante 10\% de los pacientes hipertensos). El diagnóstico de la hipertensión debe basarse en al menos tres mediciones diferentes de PA, tomadas en al menos dos visitas separadas al consultorio o al hospital. Se diagnostica hipertensión arterial cuando la PA es al menos 140 y/o 90 $\mathrm{mmHg}$. Aunque los valores obtenidos en el consultorio o en la clínica son en los que se basa usualmente el diagnóstico y el tratamiento, existen métodos adicionales de medición de la PA que son útiles en varios casos. La monitorización ambulatoria de la PA durante 24 horas (MAPA) es más relacionada con el pronóstico que la PA tomada en el consultorio $(72,73)$, y permite detectar dos subgrupos de pacientes hipertensos cuando la PA ambulatoria y la del consultorio son divergentes: la hipertensión de bata blanca (hipertensión en el consultorio y normotensión ambulatoria) y la hipertensión-enmascarada (normotensión en el consultorio e hipertensión ambulatoria). Los valores de corte para el diagnóstico de hipertensión mediante el MAPA se indican en la Tabla 5.

Hay situaciones clínicas en las que el MAPA es útil para el diagnóstico de hipertensión, por ejemplo, cuando se sospecha hipertensión de bata blanca, cuando los pacientes con hipertensión marcada no presentan signos de daño de órgano blanco y cuando se observan importantes diferencias en los valores de PA medidos en diferentes visitas.

La indicación de toma de la PA en casa se da cuando se desea aumentar la aceptación del tratamiento, que son conocidos por aumentar el cumplimiento del tratamiento. Sólo se debe utilizar dispositivos automáticos validados y el paciente debe ser instruido para realizar las mediciones en la posición sentada, después de varios minutos de reposo, idealmente en la mañana y en la tarde. Durante el tratamiento, las mediciones deben hacerse en la mañana antes de la toma de los medicamentos antihipertensivos.

En el manejo del paciente hipertenso no sólo deben considerarse los niveles de presión arterial sino también el riesgo cardiovascular global. Con el fin de estratificar el riesgo cardiovascular global debe considerarse el número

Tabla 5. Hipertensión: criterios de presión arterial.

\begin{tabular}{|ll|}
\hline Hipertensión & $\begin{array}{l}\text { Presión arterial } \geq 140 / 90 \mathrm{mmHg} \text { tomada en el consul- } \\
\text { torio o en el hospital (promedio de tres mediciones } \\
\text { visita, durante tres visitas). }\end{array}$ \\
\hline MAPA 24 h & $\geq 130 / 80 \mathrm{mmHg}$, MAPA diurno $\geq 135 / 85 \mathrm{mmHg}$ \\
\hline PA tomada en casa o auto-medición $\geq 135 / 85 \mathrm{mmHg}$ \\
\hline Hipertensión de bata blanca & $\begin{array}{l}\text { Hipertensión en el consultorio o en el hospital y normo- } \\
\text { tensión ambulatoria o en casa. }\end{array}$ \\
\hline Hipertensión enmascarada & $\begin{array}{l}\text { Normotensión en el consultorio o en el hospital con } \\
\text { hipertensión ambulatoria o en el hogar. }\end{array}$ \\
\hline
\end{tabular}


de factores de riesgo presentes, la ausencia o presencia de lesiones de órgano blanco y las condiciones clínicas previas o simultáneas, incluyendo el síndrome metabólico y la diabetes, junto con la clasificación de PA que se resumen en la Tabla 6.

\section{La hipertensión en pacientes con diabetes}

En los individuos con diabetes como resultado de la alteración de la función autonómica y el daño extenso de órganos, son comunes la presencia de una mayor variabilidad de la presión arterial, una marcada respuesta ortostática y un importante deterioro de la reducción nocturna de la PA (72). Estas características tienen implicaciones diagnósticas, pronósticas y terapéuticas. Así, se recomienda que el número de mediciones de presión arterial para la toma de decisiones debe ser mayor, la detección de hipotensión ortostática debe ser un procedimiento de rutina, y la toma de PA en el hogar y sobre todo del MAPA debe realizarse siempre que sea posible. La información actualizada sobre este tema está disponible (73) y es aconsejable la formación de los médicos en la interpretación de estos datos.

Las recomendaciones sobre la evaluación diagnóstica en los pacientes con hipertensión y diabetes se resumen en la Tabla 7. Las recomendaciones para el seguimiento son las siguientes:

1. HbA1c (cada cuatro meses).

2. Auto monitoreo de la glucosa en sangre (cada 24 a 48 horas).

3. Anualmente evaluación de fondo de ojo, ECG, microalbuminuria, pruebas básicas de laboratorio.

4. Cada dos años realizar ecocardiograma y electrocardiograma de esfuerzo (para detectar posible isquemia silente).

En términos de riesgo cardiovascular total (Tabla 6), la presencia de diabetes generalmente se considera que implica un nivel alto de riesgo, pero es razonable pensar que el riesgo cardiovascular es diferente si la diabetes es de reciente diagnóstico o si es de larga duración, en ausencia o en presencia de complicaciones. En pacientes normotensos con diabetes no hay ninguna evidencia que la administración de fármacos reductores de la PA sean de algún beneficio.

\section{Complicaciones renales y cardiovasculares en pacientes hipertensos diabéticos}

Los pacientes con diabetes e hipertensión tienen un mayor riesgo de enfermedad renal, enfermedad cardiaca coronaria (ECC), accidente cerebrovascular e insuficiencia cardíaca. La asociación con comorbilidades como la dislipidemia, estado protrombótico y disfunción autonómica (74) contribuye a aumentar la morbilidad y mortalidad.

\section{Nefropatía diabética}

La prevalencia de la nefropatía en pacientes con diabetes tipo 2 es de 30-50\% (75). Se han descrito tres etapas en su desarrollo (76):

1. Nefropatía incipiente, con una duración de unos 10 años, cursa con una tasa supranormal de filtración glomerular (TFG), que se acompaña después de unos 5 años con aumento de la excreción urinaria de albúmina (EUA: 30-300 mg/día para microalbuminuria). La presencia de un aumento de la EUA identifica a los pacientes diabéticos con alto riesgo de desarrollar daño renal progresivo y enfermedad cardiovascular.

2. Nefropatía clínica evidente, caracterizada por una EUA mayor de 300mg/día (proteinuria), TFG normal o moderadamente reducida e hipertensión. Si no se tratan, estos pacientes tienen un alto riesgo de desarrollar enfermedad renal terminal (ERT). Sin una adecuada intervención, esta condición puede progresar rápidamente, y $50 \%$ de los pacientes de pueden llegar a ERT en 10 años y $75 \%$ en 20 años. A la inversa, las intervenciones terapéuticas en ambos tipos de diabetes disminuyen la velocidad de la alteración de la TFG y la progresión a ERT y la TFG. Se ha informado que $20-40 \%$ de los individuos con EUA pueden progresar a macroalbuminuria y $20 \%$ de ellos a ERT.

Tabla 6. Estratificación del riesgo en pacientes con síndrome metabólico, hipertensión arterial y diabetes tipo 2.

\begin{tabular}{|c|c|c|c|c|c|c|}
\hline \multirow{2}{*}{$\begin{array}{l}\text { Otros factores de } \\
\text { riesgo o enfermedades }\end{array}$} & \multicolumn{3}{|c|}{ Normotensión } & \multicolumn{3}{|c|}{ Hipertensión } \\
\hline & Optima & Normal & Normal alta & Grado 1 & Grado 2 & Grado 3 \\
\hline $\begin{array}{l}\text { Sin factores de } \\
\text { riesgo (FR) }\end{array}$ & Riesgo promedio & Riesgo promedio & Riesgo promedio & Riesgo añadidobajo & Riesgo añadidomoderado & Riesgo añadido alto \\
\hline $\begin{array}{l}\text { 1-2 FR o condiciones } \\
\text { sociales de riesgo }\end{array}$ & Riesgo añadido bajo & Riesgo añadido bajo & Riesgo añadido bajo & Riesgo añadido moderado & Riesgo añadido moderado & Riesgo añadido muy alto \\
\hline $\begin{array}{l}\text { FR } \geq 3 \text { o condiciones } \\
\text { sociales de riesgo, } \\
\text { DOB o SM/DM }\end{array}$ & Riesgo añadido moderado & Riesgo añadido moderado & Riesgo añadido alto & Riesgo añadido alto & Riesgo añadido alto & Riesgo añadido muy alto \\
\hline $\begin{array}{l}\text { Condicion clínica } \\
\text { asociada }\end{array}$ & Alto riesgo añadido & Alto riesgo añadido & Riesgo muy alto & Riesgo añadido muy alto & Riesgo añadido muy alto & Riesgo añadido muy alto \\
\hline
\end{tabular}


Tabla 7. Recomendaciones de evaluación diagnóstica de los pacientes hipertensos con diabetes mellitus.

\begin{tabular}{|c|c|}
\hline & Historia clínica y el examen físico \\
\hline $\begin{array}{l}\text { Investigaciones básicas } \\
\text { o mínimas }\end{array}$ & $\begin{array}{l}\text { Medición de la presión arterial (según AAD) } \\
\text { ECG } \\
\text { Pruebas de laboratorio: glucemia en ayunas y HbA1c, } \\
\text { creatinina sérica, perfil de lípidos, enzimas hepáticas, } \\
\mathrm{Na}+, \mathrm{K}+ \\
\text { Microalbuminuria (según AAD) } \\
\text { Fondo de ojo (si es anormal interconsulta al oftalmólogo) }\end{array}$ \\
\hline Investigaciones opcionales & $\begin{array}{l}\text { MAPA } \\
\text { ECG de esfuerzo (en hombres }>40 \text { años y en mujeres } \\
\text { postmenopáusicas) } \\
\text { Ecocardiograma doppler }\end{array}$ \\
\hline
\end{tabular}

3. Insuficiencia renal progresiva que cursa con proteinuria franca ( $\geq 300 \mathrm{mg} / \mathrm{dL}$ ) y una notable reducción de la TFG $\left(<30 \mathrm{~mL} / \mathrm{min} / 1.73 \mathrm{~m}^{2}\right)$. La macroalbuminuria identifica a los pacientes diabéticos con daño renal histológico sustancial y predice un descenso lineal de la TFG.

Para la detección de la aparición y la progresión de la nefropatía diabética es obligatoria la evaluación de EUA todos los años desde el inicio de la DM2, y el cálculo de la TFG a partir de la creatinina sérica mediante el uso de una de las fórmulas actualmente validadas (Modificación de la Dieta en Enfermedad Renal, MDRD o por la fórmula del Grupo Colaborador de la Epidemiologia de la Enfermedad Renal Crónica, CKD-EPI).

\section{Enfermedad cardiaca coronaria (ECC)}

Los pacientes hipertensos con diabetes tipo 2 tienen un riesgo 1.9 veces mayor de presentar enfermedad cardiovascular que la de los pacientes hipertensos sin diabetes (77). Se considera que factores como los niveles de fibrinógeno elevados, en particular durante el control glucémico deficiente, los niveles elevados del inhibidor-1 del activador de plasminógeno y la aumentada agregación plaquetaria pueden ser los responsables (78). Estas alteraciones relacionadas con la diabetes pueden aumentar el riesgo de trombosis en el sitio de rotura de la placa y también el riesgo de reinfarto después de la terapia trombolítica o de la revascularización. Además son frecuentes las arritmias cardiacas como consecuencia de la disfunción autonómica. La evaluación de la ECC debe incluir una prueba de esfuerzo seguido, si es positivo, por un estudio de perfusión miocárdica (tomografía computarizada por emisión de fotón único).

\section{Disfunción ventricular izquierda e insuficiencia cardiaca}

La diabetes es un importante factor de riesgo para disfunción ventricular izquierda e insuficiencia cardiaca. En el estudio Mónica Glasgow la incidencia de disfunción ventricular izquierda fue mayor en los pacientes diabéticos (29\%) en comparación con los no diabéticos (7\%) (79). En el estudio de Framingham el riesgo relativo de insuficiencia cardiaca clínica fue 3.8 veces mayor en hombres diabéticos y 5.5 veces en las mujeres con diabetes en comparación con aquellos sin diabetes (80). En los pacientes diabéticos con hemoglobina glicosilada (HbA1c) menor que $7.0 \%$ la tasa de falla cardíaca fue 4.2 por 1000 pacientes-año, la misma aumentó a 9.2/1000 pacientes-año cuando la HbA1c fue mayor a 10\% (80). El mal pronóstico de estos pacientes ha sido explicado por una miocardiopatía diabética subyacente, agravada por la hipertensión y la enfermedad isquémica del corazón (81).

La alta prevalencia y las implicaciones en la morbilidad y mortalidad que tiene la insuficiencia cardíaca determina la imperiosa necesidad de la identificación temprana de sus factores de riesgo y de los signos clínicos asociados. Una historia clínica cuidadosa ayuda a detectar los síntomas de la insuficiencia cardiaca (disnea de esfuerzo, ortopnea, tos nocturna), a pesar que los pacientes con disfunción sistólica del ventrículo izquierdo pueden no presentar estos síntomas (82). Por lo tanto, el diagnóstico de la insuficiencia cardiaca en los pacientes diabéticos e hipertensos puede requerir pruebas adicionales. Aunque el electrocardiograma y la radiografía de tórax pueden ser útiles, la ecocardiografía doppler es necesaria para visualizar las alteraciones cardiacas estructurales y funcionales que subyacen a la insuficiencia cardiaca, y es la prueba recomendada cuando se sospecha de insuficiencia cardiaca. Como la insuficiencia cardiaca es un predictor de muerte cardiaca súbita, se recomienda la realización de un ECG Holter para la detección de arritmias.

\section{Accidente cerebrovascular}

Las tasas de discapacidad relacionada con ictus y accidente cerebrovascular son más altos en los diabéticos que en los no diabéticos (83). El riesgo de accidente cerebrovascular fatal versus no fatal es mayor cuanto mayor es el nivel de $\mathrm{HbA1c}$ incluso muchos años antes que se presente el evento (83-85). 


\section{Tratamiento de la hipertensión arterial en pacientes diabéticos}

\section{Tratamiento no farmacológico de la hipertensión arterial en la diabetes mellitus}

\section{Plan dietético}

El consumo de hidratos de carbono debe representar el 55-60\% de la ingesta total de calorías (ITC), minimizando los carbohidratos simples refinados (azúcar, miel, fructosa, melaza, etc.), al tiempo que se debe aumentar los carbohidratos complejos (verduras, frutas y granos enteros). El uso de edulcorantes no calóricos está permitido, pero se deben seleccionar los que tienen bajo contenido de sodio.

El consumo de proteínas debe ser de $0.8-1 \mathrm{~g} / \mathrm{Kg}$ del peso corporal ideal. Las proteínas animales son preferibles debido a su alto valor biológico, pero las legumbres y cereales deben ser incluidas para agregar a la proteína la fibra. La fibra debe consumirse en una cantidad aproximada $30 \mathrm{~g} / \mathrm{día}$, preferiblemente fibra soluble.

El consumo de grasa no debe ser mayor que $30 \%$ de la ITC, $10 \%$ debe ser saturada (grasa láctea y sus derivados), $10 \%$ poliinsaturada (aceites vegetales, frutos secos, pescado), $10 \%$ monoinsaturadas (aguacate, aceitunas, carne de cerdo, pollo).

Las recomendaciones de vitaminas y oligoelementos son similares a las que se recomienda para la población general, así el consumo de sodio debe ser de 3.2 g/día (4-6 g de cloruro de sodio), para lo cual hay que evitar los alimentos procesados. Es conveniente conocer el contenido de sodio del agua potable en la región de Latinoamérica, ya que ésta puede variar ampliamente, como acontece con el agua embotellada. Se debe realizar esfuerzos para alcanzar las recomendaciones en el consumo de calcio, sobre todo en las dietas hipocalóricas, a través de una elección adecuada de los alimentos. Es necesario considerar las circunstancias que pueden interferir con la absorción de calcio (síndrome de mala absorción, alimentos ricos en fitohemaglutininas, drogas, etc.) Las necesidades de potasio normalmente se pueden satisfacer mediante el aumento en el consumo de verduras y frutas en la dieta.

En relación al consumo de cafeína no existen evidencias consistentes de los riesgos o beneficios del consumo crónico moderado de café ( 2 tasas/día). Mientras que el consumo de alcohol está directamente relacionado con los niveles de PA y con la prevalencia de hipertensión en diferentes poblaciones. También hay pruebas de que el abuso de alcohol bloquea el efecto de los fármacos antihipertensivos. Se debe desestimular el consumo de alcohol en los pacientes diabéticos, o permitir un consumo máximo de $30 \mathrm{~g} /$ día en los hombres y $15 \mathrm{~g} /$ día en las mujeres.

La distribución de los alimentos debe hacerse en tres o cuatro comidas y en una o dos meriendas durante el día, dependiendo del horario del paciente y del tratamiento farmacológico de la diabetes mellitus, al igual que deben considerarse las preferencias étnicas, religiosas y el estatus socio-económico del paciente.

\section{Actividad física}

El estilo de vida sedentario y la falta de actividad física son fuertes predictores de mortalidad cardiovascular, independiente de la presencia de hipertensión y de otros factores de riesgo. La intensidad del ejercicio recomendado debe ser individualizada de acuerdo a la condición clínica del paciente. El examen clínico es suficiente cuando la actividad programada no supera $60 \%$ del consumo máximo de oxígeno (VO2 Max, por ejemplo, caminar). Cuando se ha previsto una actividad más intensa, es necesario un examen más extenso de las posibles complicaciones de la diabetes. Se debe prestar especial atención a la enfermedad cardiaca silente (o que está compensada en descanso), a la retinopatía proliferativa, a la nefropatía incipiente, a la enfermedad vascular periférica, a la neuropatía periférica y autonómica, y osteo-artropatía, especialmente de las extremidades inferiores, ya que el ejercicio puede contribuir a causar lesiones en los pies. Se debe preparar un programa individualizado de 3 veces a la semana, incluyendo actividad aeróbica recreativa de intensidad moderada (equivalente a 3 a 5 METS) en la forma de deporte o ejercicio en casa, que dure de 20 a 60 minutos por sesión, precedida por 5 a 10 minutos de calentamiento, y seguido por 5 a 10 minutos de relajación.

El paciente debe ser instruido sobre el uso de ropa apropiada para prevenir lesiones de los pies, tales como calcetines de algodón y zapatos deportivos. El auto monitoreo de glucosa en sangre antes y después del ejercicio puede ayudar a prevenir hipoglucemia y permitirle al paciente comprobar los efectos beneficiosos del ejercicio sobre el control glucémico (86-88). El ejercicio intenso está contraindicado en pacientes con retinopatía proliferativa activa, y con manifestaciones clínicas de nefropatía y neuropatía.

\section{Tratamiento farmacológico}

Los beneficios de la reducción de la presión arterial en los pacientes diabéticos fueron demostrados claramente en los estudios HOT (89) y UKPDS (90) entre otros (91-95). Hay que destacar que los diabéticos pueden requerir un tratamiento más intenso para alcanzar los mismos niveles de PA que los no diabéticos. En verdad, casi todos los pacientes diabéticos necesitan, además de las medidas no farmacológicas, un tratamiento hipotensor combinado para alcanzar los objetivos del tratamiento, y tan temprano como sea posible.

Recientemente ha sido ampliamente debatida la meta de PAS que debe alcanzarse para garantizar una protección óptima del riesgo cardiovascular en pacientes hipertensos con diabetes. Una serie de directrices en el pasado $(1,2,69$, 
70) habían recomendado una meta menor $(<130 / 80 \mathrm{mmHg})$ en diabéticos y en general en pacientes de alto riesgo) a la recomendada $(<140 / 90 \mathrm{mmHg})$ en pacientes hipertensos de riesgo bajo-moderado, sin embargo una revalorización reciente de la evidencia disponible $(71,96)$ demostró que ninguno de los ensayos clínicos aleatorios de tratamiento antihipertensivo en diabéticos con hipertensión logró un promedio de los valores de PAS por debajo de $130 \mathrm{mmHg}$, y el reciente estudio Action to Control Cardiovascular Risk in Diabetes (ACCORD) (97) no mostró ninguna reducción adicional de los eventos cardiovasculares, y más bien se observó una mayor incidencia de efectos adversos en los pacientes diabéticos aleatorizados para alcanzar un PAS $<120 \mathrm{mmHg}$ en comparación con aquellos aleatorizados para alcanzar una PAS $<140 \mathrm{mmHg}$ (los valores medios realmente alcanzados fueron de $119 \mathrm{mmHg}$ y $133 \mathrm{mmHg}$ ). Una serie de recientes meta análisis $(98,99)$ dirigidos a correlacionar los eventos cardiovasculares con la PA obtenida, no han encontrado ningún beneficio adicional o un empeoramiento de la incidencia de eventos cardiovasculares cuando se alcanza una presión arterial más baja, con la posible excepción de la incidencia de accidentes cerebrovasculares (99). En realidad, parece que en pacientes hipertensos con diabetes el objetivo de PAS $<140 \mathrm{~mm} \mathrm{Hg}$ debe ser recomendado al igual que en los no diabéticos hipertensos. Los valores apenas por encima de $130 \mathrm{mmHg}$ (como se logró en ACCORD (97) y ADVANCE (100)) parece ser segura y puede ser más eficaz en la reducción o prevención de microalbuminuria (100). En cuanto al objetivo de PAD, los resultados del HOT (89) y del UKPDS (90) indican que los valores entre 80 y $85 \mathrm{mmHg}$ son beneficiosos.

En cuanto a los pacientes diabéticos con nefropatía, las guías anteriores recomendaron como objetivo de presión arterial cifras de $<130 / 80 \mathrm{mmHg}$ y $<120 / 75$ en caso de proteinuria. Una revisión reciente (101) demostró que estas recomendaciones no se basaron en resultados de ensayos clínicos de largo plazo, y que se obtuvieron de estudios observacionales no aleatorizados. Parece prudente por lo tanto, recomendar que las metas de presión arterial sean las mismas para los pacientes diabéticos con y sin nefropatía.

Cinco clases de agentes antihipertensivos (diuréticos, bloqueadores beta, inhibidores de la enzima convertidora de angiotensina (IECA), antagonistas de los receptores de angiotensina II (ARA) y bloqueadores de canales de calcio (BCC)) han sido utilizados en los ensayos clínicos aleatorizados que demostraron que reducir la presión arterial reduce significativamente los eventos cardio-cerebro-vasculares, y la afectación renal en pacientes hipertensos con diabetes y sin diabetes (102). Por lo tanto, todos ellos pueden ser utilizados en pacientes hipertensos con diabetes tipo 2 . Sin embargo se recomienda que para iniciar el tratamiento con monoterapia se debe elegir los fármacos que bloquean el sistema renina-angiotensina-aldosterona (IECAs o ARAs) debido a su mayor efecto antiproteinúrico. Los ARAs son generalmente mejor tolerados, tema que es relevante en pacientes con hipertensión y diabetes en los cuales la adherencia al tratamiento es esencial. Como regla general se recomienda seleccionar un agente de acción prolongada que proporciona reducción de la presión arterial durante las 24 horas con el fin de utilizar una administración diaria única. Las agencias reguladoras norteamericana y europea (FDA y EMEA) han aprobado a ramipril (IECA) y a telmisartán (ARA) como fármacos cardioprotectores en pacientes con alto riesgo cardiovascular (pacientes hipertensos con diabetes tipo 2) con base en los resultados de los estudio HOPE (94) y ONTARGET (103).

En la mayoría de los pacientes hipertensos con diabetes tipo 2 no es fácil alcanzar las metas de presión arterial con monoterapia por lo que el tratamiento debe incluir dos o más agentes antihipertensivos. Si antes del tratamiento la PAS/PAD están muy lejos de los valores objetivo, se recomienda iniciar el tratamiento con una combinación de dos medicamentos, una combinación fija de un IECA o ARA con un BCC dihidropiridínicos o un diurético. El estudio The Avoinding Cardiovascular Eventstrough Combination Therapy in Patients Living with Systolic Hypertension (ACCOMPLISH) (104) demostró mayores beneficios con un IECA/CCB en lugar de una combinación IECA/diurético, pero estos interesantes datos deben ser confirmados. Cuando se necesitan tres medicamentos, se recomienda utilizar un IECA o un ARA más un CCB y un diurético tiazídico. En pacientes con una $\mathrm{TFG}<30 \mathrm{ml} / \mathrm{min}$, los diuréticos tiazídicos debe ser sustituido por un diurético de asa (como furosemida) en las dosis apropiadas.

Los diuréticos y los beta bloqueadores, particularmente en asociación, aumentan la resistencia a la insulina y pueden facilitar la aparición de diabetes en sujetos predispuestos, por lo que ésta asociación se debe evitar en los posible en pacientes hipertensos con pre-diabetes o con síndrome metabólico. Recientemente se ha demostrado que los beta bloqueadores vasodilatadores, tales como nebivolol y carvedilol, no afectan la sensibilidad a insulina, y en el caso de nebivolol se ha demostrado que no altera la tolerancia a la glucosa, incluso en asociación con diuréticos tiazídicos (106). Por lo tanto, los beta bloqueadores vasodilatadores deben ser preferidos en aquellas condiciones en las cuales hay razones de peso para la administración de un bloqueador beta (enfermedad cardíaca isquémica, insuficiencia cardíaca, taquiarritmias, etc).

En pacientes con insuficiencia renal y/o disfunción cardíaca, la función cardiaca puede mejorar mediante la administración de antagonistas del receptor de mineralocorticoides (espironolactona, eplerenona), los cuales se han demostrado eficaces en la hipertensión resistente. Sin embargo, los niveles séricos de potasio y la TFG deben ser estrechamente controlados en los pacientes con enfermedad renal utilizando un inhibidor del sistema RAA y un antagonista de aldosterona.

Se ha demostrado que los bloqueadores alfa mejoran la resistencia a la insulina y podrían utilizarse como un agente 
adicional en pacientes hipertensos con diabetes tipo 2 en los cuales no se alcanzan los objetivos de presión arterial, sin embargo estos agentes no se recomiendan como monoterapia excepto en pacientes hipertensos con hipertrofia prostática. La Tabla 8 indica los fármacos antihipertensivos que deben ser preferidos para el manejo farmacológico de los pacientes hipertensos con diabetes tipo 2 y condiciones especiales.

\section{Poblaciones especiales}

\section{Hipertensión y diabetes en los afro-latinoamericanos}

La población latinoamericana está constituida por diferentes etnias (107). La prevalencia de los diferentes grupos étnicos en cada país de América Latina se caracteriza por una mezcla de razas, etnias y culturas, como en ningún otro continente.

A pesar de la gran cantidad de población negra en América Latina, no existe ningún estudio epidemiológico sobre la prevalencia de hipertensión y diabetes en esta población y ningún estudio ha investigado, en una muestra suficientemente grande y utilizando metodologías consistentes, la ingesta de alimentos, la actividad física, la composición corporal asociadas con hipertensión y diabetes. La mayor parte de la información obtenida resulta de los estudios realizados en los EE.UU. que incluyeron a población negra que ha emigrado de América Latina y el Caribe a EE.UU. $(108,109)$ o jóvenes negros nacidos en EE.UU. pero de origen latino y del Caribe (32).

Por lo tanto, la primera recomendación del Consenso Latinoamericano es la de apelar para que las organizaciones académicas y gubernamentales en América Latina apoyen la investigación epidemiológica, clínica y terapéutica en los afrodescendientes que habitan en Latinoamérica para comprobar si los resultados de los estudios de EE.UU. también se aplican a la población negra que vive en América Latina. Al momento existe un único estudio que evalúa la importancia de la hipertensión arterial en un distrito rural de negros que viven en la provincia de Esmeraldas en Ecuador (110), en donde se examinaron 4284 de los 8876 adultos que viven en la zona. 1542 (36\%) fueron hipertensos, y de los cuales sólo cuatro $(0.3 \%)$ estaban bien controlados con el tratamiento recibido. En los 2.5 años de seguimiento, las enfermedades cardiovasculares fueron la principal causa de muerte en la población adulta. Además, cuatro de cada cinco personas que murieron por ECV, tenían antecedentes de hipertensión arterial. En verdad, la prevalencia de hipertensión no controlada en este estudio fue mucho mayor que lo reportado en los estudios realizados en EE.UU.

Hasta no tener una cantidad adecuada de datos originados de estudios que deben realizarse en la población negra de América Latina, el Consenso recomienda la adopción de las recientes guías de la Sociedad Internacional de Hipertensión en negros (111). De acuerdo con este último documento existe una clara diferencia geográfica en la prevalencia de la hipertensión entre personas de raza negra, así: 14\% en África Occidental, $26 \%$ en el Caribe y el $33 \%$ en EE.UU. Estas diferencias son tentativamente atribuidas a diferencias en la dieta y en el estilo de vida. En Estados Unidos las mujeres negras son más sedentarias, tienen un elevado aporte calórico y son más obesas desde el período pre-adulto $(112,113)$. Los factores genéticos y ambientales, tales como el bajo nivel socio-económico, la alta ingesta de sodio en la dieta y/o baja de potasio, y el peso bajo al nacer por desnutrición materna, han sido asociados con un pobre desarrollo renal y menor número de nefronas, lo que predispone a hipertensión arterial y la disfunción renal temprana $(114,115)$.

Las complicaciones cardiorenales relacionadas con la hipertensión arterial y la diabetes tipo 2 (derrame cerebral, hipertrofia ventricular izquierda, insuficiencia cardíaca crónica en fase terminal o insuficiencia renal) se producen más a menudo en negros que en blancos. Los negros hipertensos tienen un riesgo de 4 a 20 veces mayor de progresión a diálisis que los blancos con similares niveles de presión arterial, y la mortalidad en los hombres afroamericanos es tres veces mayor $(49 \%)$ que en los blancos no hispanos en EE.UU. (16\%), y dos veces y media mayor en mujeres de raza negra (37\%) que en las mujeres blancas no hispanas (14\%) (116).

Tabla 8. Recomendaciones con otros medicamentos de uso para los pacientes hipertensos con tipo diabetes 2 y las condiciones especiales.

\begin{tabular}{|l|l|}
\hline $\begin{array}{l}\text { Enfermedad coronaria y/o } \\
\text { disfunción ventricular izquierda. }\end{array}$ & $\begin{array}{l}\text { IECA/ARA, beta-bloqueadores, antagonistas de } \\
\text { la aldosterona }\end{array}$ \\
\hline $\begin{array}{l}\text { Hipertensión sistólica aislada } \\
\text { en los ancianos }\end{array}$ & $\begin{array}{l}\text { Bloqueadores de los canales de calcio, diuréticos, } \\
\text { ARA }\end{array}$ \\
\hline Angina de pecho & $\begin{array}{l}\text { Bloqueadores de los canales de calcio, beta- } \\
\text { bloqueadores, a menudo asociados }\end{array}$ \\
\hline Enfermedad renal crónica & $\begin{array}{l}\text { IECA o ARA, sobre todo en presencia de micro- } \\
\text { albuminuria o proteinuria franca }\end{array}$ \\
\hline Enfermedad arterial periférica & Bloqueadores de los canales de calcio \\
\hline Pacientes con fibrilación auricular & $\begin{array}{l}\text { Bloqueadores beta, ARA, IECA, bloqueadores } \\
\text { de los canales del calcio no dihidropiridínicos }\end{array}$ \\
\hline Hipertrofia ventricular izquierda & IECA, ARA, BCC \\
\hline Hipertrofia prostática benigna & Bloqueadores alfa \\
\hline
\end{tabular}


La elección entre monoterapia antihipertensiva o terapia combinada depende de la presencia o ausencia de comorbilidades, y de la eficacia específica de los medicamentos a ser utilizados. Estudios comparativos han demostrado que los hipertensos negros tienen una mejor respuesta a los diuréticos tiazídicos (hidroclorotiazida o clortalidona) y a los bloqueadores de los canales de calcio, que a los IECA o los ARA, o la los bloqueadores beta $(117,118)$. El mejor control siempre se obtiene si se reduce la ingesta de sodio. Además, los negros son más propensos a presentar edema angioneurótico en respuesta a los inhibidores de la ECA que los blancos (119). Por lo tanto, en los negros la monoterapia debe basarse en un diurético o un bloqueador de los canales de calcio, y cuando se requiera de la terapia combinada esta debe incluir un BCC y/o un diurético más un bloqueador del sistema RAA, preferiblemente un ARA.

\section{Hipertensión y diabetes en la población Andina}

La población de América Latina que vive en la Cordillera de los Andes comparte similares características y patrones históricos de colonización con los que viven en altitudes más bajas, siendo en su mayoría Amerindios o mestizos. Las personas que viven a gran altitud (más de $3000 \mathrm{msnm}$ ) constituyen un grupo especial en el que la prevalencia de la hipertensión y diabetes es muy poco conocida. Un estudio poblacional (120) que incluyó 1878 adultos en los Andes peruanos, mostró que la prevalencia de hipertensión fue del 15.7\% (95\% intervalo de confianza IC: $14.0-17.4 \%$ ), que no hubo diferencias por sexo, $y$ que aumentó considerablemente con la edad, sobre todo en las mujeres. Las tasas de conocimiento, tratamiento y control fueron de $47.9 \%, 39.5 \%$, y $14 \%$, respectivamente. La presión arterial diastólica aumentó hasta la edad de 50 años y alcanzó una meseta a partir de entonces, mientras que la presión arterial media siguió aumentando con la edad, incluso después de 50 años de edad.

El tipo predominante de hipertensión fue sistodiastólica $(41.7 \%$, IC 95\%, 35.1-48.5\%) o diastólica aislada. La hipertensión sistólica aislada representó sólo el 29.3\% de los casos (95\% IC, 23.9-35.4\%) y fue responsable de una minoría de casos en todos los grupos de edad antes de los 70 años. El dato que la hipertensión diastólica es la predominante en la Cordillera de los Andes a más de 3000 msnm fue recientemente confirmado en otro estudio (121), en el que se encontró que más de 50\% de esta población no conocía de su condición de hipertenso. Este estudio también mostró que la prevalencia de hipertensión arterial fue similar en la costa, sierra y selva del Perú $(120,121)$.

\section{Hipertensión y diabetes en los ancianos}

El reporte demográfico de la Organización Panamericana de la Salud (OPS)/WHO en Latinoamérica (122) demuestra que la población mayor de 60 años representa el 14\% de la población total en Argentina, el 10\% en Brasil, el $13 \%$ en Chile, el $8 \%$ en Colombia, el 9\% en Ecuador, el $7 \%$ en Paraguay, el $8 \%$ en Perú, el $18 \%$ en Uruguay, el $8 \%$ en Venezuela y el 8\% en México.

Los ancianos, definidos como las personas mayores de 65 años, tienen un mayor riesgo de hipertensión arterial, especialmente de hipertensión sistólica aislada $(123,124)$ lo que implica un riesgo cardiovascular adicional, ya que la presión de pulso superior a $65 \mathrm{mmHg}$ se asocia con una mayor rigidez de la pared de las arterias grandes y aumentada morbilidad y mortalidad cardiovascular (124). El MAPA durante 24 horas se considera una herramienta útil para optimizar la evaluación clínica de los pacientes hipertensos de edad avanzada $(125,126)$, en quienes se observa una caída nocturna anormal de la PA y oleadas de aumento matutino de la PA comportamiento que se asocia con mayor riesgo de enfermedad cerebrovascular $(127,128)$, aunque estos hallazgos han sido recientemente cuestionados (129).

Todos los ensayos que han demostrado los beneficios de la disminución de la PA en los ancianos se han dirigido a un objetivo de PAS $<150 \mathrm{mmHg}$ (96), y éste debe ser considerado como el objetivo meta basado en la evidencia para ancianos hipertensos, pero por otro lado en ancianos sanos se recomienda un objetivo similar a la propuesta para hipertensos jóvenes $(\leq 140 \mathrm{mmHg})$. También hay evidencia de beneficios en la reducción de la PAS a $<150 \mathrm{mmHg}$ en hipertensos mayores de 80 años (130). Personas frágiles o complicadas deben ser tratadas con especial atención para no empeorar sus condiciones generales de salud.

En las personas de edad avanzada el tratamiento farmacológico debe iniciarse gradualmente para garantizar una buena tolerabilidad y calidad de vida. La sexualidad (disfunción sexual), el sueño y el estado funcional deben ser considerados en la evaluación clínica de esta población (10).

Diversos ensayos clínicos han demostrado los beneficios de la reducción de la hipertensión sistólica aislada (131-133), mediante el uso de diuréticos o BCC. Otros ensayos clínicos en ancianos hipertensos en los cuales se incluyó un número importante de pacientes con hipertensión sistólica aislada, han utilizado IECAs y ARAs, fármacos que también son útiles en las personas de edad avanzada, tanto en monoterapia como en combinación.

En aquellos pacientes con riesgo cardiovascular asociado o comorbilidades, el fármaco de elección debe ser seleccionado de acuerdo con la enfermedad concomitante de acuerdo a lo indicado en la Tabla 8. Son recomendables las drogas de larga acción para obtener un mejor cumplimiento y una acción antihipertensiva sostenida de 24 horas. 


\section{El papel de medio ambiente y la epigenética en el síndrome metabólico, la hipertensión y la diabetes en América Latina}

El aumento en la incidencia de síndrome metabólico, diabetes tipo 2 y enfermedades cardiovasculares en América Latina parece estar asociado a las influencias ambientales y a las características étnicas de la población (134). Esto plantea la posibilidad que la predisposición genética asociada a determinados grupos étnicos puede interactuar con factores ambientales para explicar las diferencias en la incidencia de la enfermedad. Recientemente existe un considerable interés en la especial influencia de la exposición medioambiental durante la vida uterina y en la vida extrauterina temprana. La propuesta se basa en la hipótesis de los orígenes evolutivos de la enfermedad la cual enfatiza que las condiciones ambientales durante los períodos críticos de la vida temprana en las que se estructura el organismo y la función corporal se establecen de por vida. Más recientemente, los primeros efectos del medio ambiente se han concebido en términos de la epigenética.

La epigenética es la ciencia que explica la variación de la expresión génica en respuesta a cambios en las condiciones ambientales. Este término incluye cualquier proceso que altera la actividad del gen sin cambiar la secuencia de ADN y conduce a modificaciones rápidas pero reversible del ADN (por ejemplo, metilación) o alteraciones en la histona de la cromatina, modificaciones que pueden ser transmitidas a las células hijas. La metilación del ADN en una región reguladora de un gen específico puede inhibir la expresión génica. La cromatina es el complejo nuclear que consiste de ADN envuelto alrededor de las proteínas histonas que pueden ser modificadas por acetilación e influir en la expresión de los genes (135).

Los mecanismos que controlan los procesos epigenéticos todavía no están completamente comprendidas, pero sí está claro que las variaciones hereditarias del ADN podrían alterar la sensibilidad a ciertos desencadenantes ambientales o cambiar la naturaleza de las respuestas epigenéticas para una determinada exposición. En el contexto Latinoamericano, la cuestión es determinar si las diferencias en la sensibilidad para desarrollar síndrome metabólico a menores niveles de grasa visceral es dependiente de la variación regional y étnica en los procesos epigenéticos o simplemente se deben a diferencias en la exposición ambiental.

Es bien conocido que en América Latina, la desnutrición materna y la desnutrición infantil son un problema importante que aún no se han resuelto en una importante proporción de las poblaciones pobres (136). Además, en América Latina se ha encontrado una alta prevalencia de hipertensión arterial en los niños, adolescentes y adultos con retraso en el crecimiento por una mala nutrición (137-144). Un estudio realizado en Brasil (137), que investigó el comportamiento de la presión arterial en una muestra aleatoria de adolescen- tes con retraso en el crecimiento $(10-16 y, n=56)$, residentes en tugurios mostraron un elevado porcentaje de personas con una presión arterial por encima de los percentiles 90 y 95, luego de ajustar por la estatura, y estuvieron en mayor riesgo de desarrollar hipertensión. Considerando al grupo de pacientes como un todo, la prevalencia de hipertensión arterial diastólica fue $21 \%(95 \% \mathrm{CI}=10-32 \%)$. La prevalencia de los casos con una presión sistólica o diastólica arterial por encima del percentil 90 fue de $51 \%$ (95\% CI= $37-65 \%)$. Otro estudio realizado en el nordeste de Brasil (138) en 416 adultos (18-60 años), también residentes de un barrio pobre, mostró que la prevalencia de hipertensión arterial fue de $28.5 \%$ (mujeres $=38.5 \%$; hombres $=18.4 \%$ ). La presión arterial sistólica y diastólica aumentó de acuerdo con la menor estatura, y la hipertensión fue más frecuente en las mujeres que fueron obesas y de baja de estatura (50\%) en relación con las obesas pero que no eran pequeñas $(\mathrm{OR}=$ $1.98, \mathrm{IC}=1.22-2.96$ ).

Recientemente, otro estudio (139) investigó si las condiciones de salud de las madres que tenían una estatura baja eran diferentes a la de aquellas mujeres sin desnutrición, o a la de sus hijos. La baja estatura materna se asoció de forma independiente con obesidad, obesidad abdominal y presión arterial elevada. Además, la baja estatura materna se asoció con bajo peso al nacer y retraso del crecimiento en los niños nacidos de estas madres. En Colombia se demostró que la presión arterial de niños de 11 años de edad que tenían una media de IMC de $21 \mathrm{Kg} / \mathrm{m}^{2}$, el más alto tertil, presentó un incremento de $10 \mathrm{mmHg}$ en relación con los niños con un IMC medio de $15 \mathrm{Kg} / \mathrm{m}^{2}$, el tertil inferior (140). Franco et al. en Brasil, (141) reportaron cambios en los sistemas simpático-adrenal y renina-angiotensina en niños pequeños para su edad gestacional. Ellos investigaron los niveles plasmáticos de enzima convertidora de angiotensina (ACE), de angiotensina y de catecolaminas en niños de 8 a 13 años de edad, y determinaron las correlaciones entre los niveles plasmáticos, el peso al nacer yla presión arterial. Los niveles circulantes de noradrenalina estuvieron significativamente elevados en niñas pequeñas para la edad gestacional en comparación con las niñas que nacieron con un peso adecuado para su edad gestacional. Además, la angiotensina II y la actividad de la ECA fueron más altos en los niños pequeños para la edad gestacional. Además se observó una asociación significativa entre los niveles circulantes de angiotensina II y la actividad de ECA y PAS. Otro estudio en Brasil (142) mostró que la actividad de la ACE se incrementa junto con un aumento de la presión sistólica y diastólica en niños con retraso del crecimiento, independiente del peso al nacer.

A pesar de que en América Latina la prevalencia de diabetes mellitus tipo 2 en individuos con bajo peso al nacer y 
con desnutrición en sus primeros años de vida no se conoce, si sabemos que los países pobres con un acelerado proceso de urbanización son particularmente vulnerables y han experimentado un incremento considerable en la prevalencia de diabetes (143). Se han reportado cambios perjudiciales en el metabolismo de la glucosa en niños mexicanos que sufren de desnutrición en la infancia. El estudio examinó los efectos de la desnutrición en el primer año de vida en la tolerancia a la glucosa y la insulina plasmática y se encontró que la desnutrición a inicios de la vida extra-uterina, independiente del peso al nacer, se asoció con hiperinsulinemia y una sensibilidad reducida a la insulina, lo cual empeoró a medida que el IMC aumentó en la vida adulta (143).

En realidad es interesante especular que el aumento en las prevalencias de hipertensión arterial, síndrome metabólico y diabetes tipo 2 que se observa actualmente en América Latina pueda ser el resultado de la discrepancia entre el medio ambiente nutricional durante la vida fetal y la primera infancia y el medio ambiente nutricional y de estilos de vida du- rante la etapa adulta. Esta discrepancia causa una confusión entre la programación fetal del sujeto y las circunstancias del adulto creadas por la imposición de los nuevos estilos de vida (144). El conflicto entre la programación fetal y la presencia tardía de obesidad abdominal puede producir una mayor sensibilidad de nuestra población para desarrollar un estado de inflamación de bajo grado, resistencia a la insulina y consecuentemente una epidemia de hipertensión, síndrome metabólico y diabetes. Los papeles relativos jugados por los factores genéticos y medioambientales y la interacción entre los dos es todavía materia de gran debate que amerita clarificar con investigaciones futuras.

La recomendación del Consenso Latinoamericano es la de estimular a la academia para desarrollar investigación orientada a establecer los mecanismos epigenéticos que explican la relación entre la malnutrición materna, la restricción del crecimiento temprano y la aparición posterior de obesidad abdominal y enfermedad cardiovascular en América Latina.

\section{Agradecimientos Participantes del Consenso}

\author{
Directores \\ LÓPEZ-JARAMILLO, Patricio (Colombia) \\ SÁNCHEZ, Ramiro (Argentina)

ACCINI, Jose Luis (Colombia)
ALVERNIA, Sergio (Colombia)
ARCOS, Edgar (Colombia)
AYALA, Myrian (Paraguay)
BENDERSKY, Mario (Argentina)
BOLÍVAR, Fabio (Colombia)
BOTERO, Rodrigo (Colombia)
BRYCE, Alfonso (Perú)
BUELVAS, Janes (Colombia)
CALDERÓN, Carlos (Colombia)
CÁRDENAS, Juan Mauricio (Colombia)
CASANOVA, María Eugenia (Colombia)
CASTILLO, Gilberto (Colombia)
COBOS, Leonardo (Chile)
CURE, Carlos (Colombia)
DÍAZ, Margarita (Uruguay)
DUARTE, Yan Carlos (Ecuador)
DUPERLY, John (Colombia)
ECHEVERRÍA, Luis (Colombia)
ESPINOSA, Tatiana (Colombia)
FELICIANO, John (Colombia)
FOSS, Milton C (Brasil)
FREIRE, Peggy (Ecuador)
GARCÍA, Henry (Colombia)
GARCÍA, Luis Hernando (Colombia)

\author{
Coordinadores \\ RAMÍREZ, Agustín J (Argentina)
}

SCHMID, Helena (Brasil)

\author{
Asesores \\ ZANCHETTI, Alberto (Italy) \\ HARRAP, Stephen (Australia)
}

\section{Participantes}

GARCÍA, Santiago (Ecuador)

GÓMEZ-ARBELÁEZ, Diego (Colombia)

HERNÁNDEZ, Erick (Colombia)

HIGUERA, Juan D (Colombia)

HUERTAS, Diego (Colombia)

JARAMILLO, Sergio (Colombia)

JÁUREGUI, Isabel (Colombia)

LANAS, Fernando (Chile)

LARA, Joffre (Ecuador)

LIZCANO, Fernando (Colombia)

MACHADO, Livia (Venezuela)

MANRIQUE, Helard (Peru)

MANZUR, Fernando (Colombia)

MÁRQUEZ, Álvaro (Colombia)

MÁRQUEZ, Gustavo (Colombia)

MARTÍNEZ, Javier (Colombia)

MARTÍNEZ, Luz X (Colombia)

MEDINA, Félix (Perú)

MEDINA, Roberto (México)

MELGAREJO, Enrique (Colombia)

MERCHÁN, Alonso (Colombia)

MIRANDA, Harold (Colombia)

MOLINA, Dora I (Colombia)

NAVARRETE, Solón (Colombia)

PARRA, Gustavo (Colombia)

\author{
PARRA CARRILLO, José Z (México) \\ PASQUEL, Miguel (Ecuador) \\ PEÑA, Jesús Alirio (Colombia) \\ PEÑAHERRERA, Ernesto (Ecuador) \\ PEREZ, Maritza (Colombia) \\ PINEDA, Belkis (Colombia) \\ PISKORZ, Daniel (Argentina) \\ PONTE, Carlos (Venezuela) \\ PRAT, Hernán (Chile) \\ REY, Juan José (Colombia) \\ RODRIGUEZ, Jesús (Colombia) \\ RODRIGUEZ, Patricia (Colombia) \\ SÁNCHEZ, Gregorio (Colombia) \\ SIERRA, Iván D (Colombia) \\ SOTOMAYOR, Arístides (Colombia) \\ SYNAY, Isaac (Argentina) \\ URIBE, Juan Carlos (Colombia) \\ URINA, Manuel (Colombia) \\ VARGAS, Ricardo (Chile) \\ VESGA, Boris (Colombia) \\ VELANDIA, Carlos (Colombia) \\ VILLAR, Raúl (Chile) \\ VILLARREAL, Eduardo (Colombia) \\ YENES, Alejandro (Chile)
}




\section{Conflicto de Interés}

No existen conflictos de interés

\section{Referencias}

1. World Health Organization, International Society of Hypertension Writing Group. 2003 World Health Organization (WHO)/International Society of Hypertension (ISH) statement on management of hypertension. J Hypertens 2003; 21: 1983-1992.

2. European Society of Hypertension-European Society of Cardiology Guidelines Committee. 2003 European Society of Hypertension-European Society of Cardiology guidelines for the management of arterial hypertension. J Hypertens 2003; 21: 1011-1053.

3. Delamothe T. Wanted: guidelines that doctors will follow. BMJ 1993; 307(6898): 218.

4. McColl A, Smith H, White P, Field J. General practitioners' perceptions of the route to evidence based medicine: a questionnaire survey. $B M J 1998 ; \mathbf{3 1 6}$ : 361-365.

5. Woolf SH. Practice guidelines, a new reality in medicine. II. Methods of developing guidelines. Arch Intern Med 1992; 152: 946-952.

6. Cabana MD, Rand CS, Powe NR, Wu AW, Wilson MH, Abboud PA, Rubin HR. Why don't physicians follow clinical practice guidelines? A framework for improvement. JAMA 1999; 282(15): 1458-1465.

7. World Health Organization. Preventing chronic diseases: a vital investment: WHO global report. Geneva: World Health Organization; 2005. Page 1-13. (cited 2012 Sep 10). Available from: http://www.who.int/chp/chronic_disease_report/ en/

8. Organización Panamericana de la Salud.Las Américas: una población creciente urbana que está envejeciendo. Washington: OPS; 2002. (cited 2012 Sep 10). Available from: http://www.paho.org/Spanish/DBI/MDS/Press1_SEA_2002, $\mathrm{htm}$

9. Organización Panamericana de la Salud. Salud en las Américas 2007. Washington: OPS; 2007. (cited 2012 Sep 10). Available from: http://www.paho.org/ hia/home.html

10. Sánchez RA, Ayala M, Baglivo H, Velázquez C, Burlando G, Kolmann O, et al. On behalf of the Latin American expert Group. Latin American guidelines on Hypertension. J Hypertens 2009; 27: 905-922.

11. Ezzati M, Lopez AD, Rodgers A, Van der Hoorn S, Murray CJ.Comparative Risk Assessment Collaborating Group.Selected major risk factors and global and regional burden of disease. Lancet 2002; 360: 1347-1360.

12. Schargrodsky H, Hernández-Hernández $\mathbf{R}$, Champagne BM, Silva $\mathbf{H}$, Vinueza R, Silva-Ayçaguer LC, et al. for the CARMELA study. CARMELA: assessment of the CV risk in seven Latin American cities. Am J Med 2008; 121: 58-65.

13. Barreto S, Azeredo V, Oliveira J, Guerra H, Guati-Mosim P, Furtado M. Hypertension and clustering of cardiovascular risk factors in a community in southeast Brazil. The Bambuí Health and Ageing Study. Arq Bras Cardiol 2001; 77: 576-581.

14. Jiménez J, Palacios M, Cañete F, Barriocanal L, Medina U, Figueredo R, et al. Prevalence of diabetes mellitus and associated cardiovascular risk factors in an adult urban population in Paraguay. Diabetic Medicine 1998; 15: 334-338.

15. Fasce E, Campos I, Ibañez P, Flores M, Zarate H, Román O, Fasce F. Trends in prevalence, awareness, treatment and control of hypertension in urban communities in Chile. J Hypertens 2007; 25: 1807-1811.

16. Guerrero-Romero F, Rodríguez M. Prevalencia de hipertensión arterial y factores asociados en la población rural marginada. Salud Pública México 1998; 40: $339-346$.

17. Teo K, Chow CK, Vaz M, Ranjarajan S, Yusuf S. PURE Investigators-Writing Group. The Prospective Urban Rural Epidemiology (PURE) study: examining the impact of societal influences on chronic non-communicable diseases in low-, middle-, and high-income countries. Am Heart J 2009; 158: 1-7.

18. Sempértegui F, Estrella B, Tucker KL, Hamer DH, Narvaez X, Sempértegui $\mathbf{M}$, et al. Metabolic syndrome in the elderly living in marginal peri-urban communities in Quito, Ecuador. Public Health Nutr 2011; 14: 758-767.

19. Alvarez C, Salazar R, Galindez J, Rangel F, Castañeda ML, Lopardo G, et al. Metabolic syndrome in HIV-infected patients receiving antiretroviral therapy in Latin America. Braz J Infect Dis 2010; 14: 256-263.

20. Bermúdez V, Marcano RP, Cano C, Arráiz N, Amell A, Cabrera M, et al. The Maracaibo city metabolic syndrome prevalence study: design and scope. Am J Ther 2010; 17: 288-294.

21. Escobedo J, Schargrodsky H, Champagne B, Silva H, Boissonnet CP, Vinueza R, et al. Prevalence of the metabolic syndrome in Latin America and its association with sub-clinical carotid atherosclerosis: the CARMELA cross sectional study. Cardiovasc Diabetol 2009; 26: 8-52.

22. Caceres M, Teran CG, Rodriguez S, Medina M. Prevalence of insulin resistance and its association with metabolic syndrome criteria among Bolivian children and adolescents with obesity. BMC Pediatr 2008; 8: 31.

23. Royer M, Castelo-Branco C, Blümel JE, Chedraui PA, Danckers L, Bencosme A, et al. Collaborative Group for Research of the Climacteric in Latin America. The USA National Cholesterol Education Programme Adul Treatment Panel III (NCEP ATP III): prevalence of the metabolic syndrome in postmenopausal Latin American women. Climacteric 2007; 10: 164-170

24. Bustos P, da Silva AA, Amigo H, Bettiol H, Barbieri MA. Metabolic syndrome in young adults from two socio-economic Latin American settings. Nutr Metab Cardiovasc Dis. 2007; 17: 581-589.

25. Rueda-Clausen C, Silva F, López-Jaramillo P. Epidemic of obesity and overweigh in Latin America and the Caribbean. Int J Cardiol 2008; 123: 111-112.

26. Garcia RG, Pérez M, Maas R, Schwedhelm E, Böger RH,López-Jaramillo P. Plasma Concentrations of Asymmetric Dimethylarginine (ADMA) in Metabolic Syndrome. Int J Cardiol 2007; 122: 176-178.

27. López-Jaramillo P, Rueda-Clausen C, Silva FA. The utility of differen definitions of metabolic syndrome in Andean population. Int J Cardiol 2007; 116: $421-422$.

28. Garcia RG, Cifuentes AE, Caballero RS, Sánchez L, López-Jaramillo P. A proposal for an appropriate central obesity diagnosis in Latin American population. Int J Cardiol 2005; 110: 263-264

29. Pérez M, Casas JP, Cubillos LA, Serrano NC, Silva FA, Morillo CA, et al Using waist circumference as screening tool to identify Colombian subjects a cardiovascular risk. Eur J Cardiovasc Preven and Rehab 2003; 10: 328-335.

30. Pinzón JB, Serrano NC, Díaz LA, Mantilla G, Velasco HM, Martínez $\mathbf{L X}$, et al. Impacto de las nuevas definiciones en la prevalencia de síndrome metabólico en una población de Bucaramanga, Colombia. Biomédica 2007 27: 172-179.

31. Piegas LS, Avenzum A, Pereira JC, Neto JM, Hoepfner C, Farran JA, et al Risk factors for myocardial infarction in Brazil. Am Heart J 2003; 146: 331-338

32. Messiah SE, Carrillo-Iregui A, Garibay-Nieto N, López-Mitnik G, Cossio S, Arheart KL. Prevalence of metabolic syndrome in US-born Latin and Caribbean youth. J Immigr Minor Health 2009; 11: 366-371.

33. Velásquez-Meléndez G, Kac G, Valente JG, Tavares R, Silva CQ, Garcia ES. Evaluation of waist circumference to predict general obesity and arteria hypertension in women in Greater Metropolitan Belo Horizonte, Brazil. Cad Saude Publica 2002; 18: 765-771.

34. Berber A, Gómez Santos R, Fanghanel G, Sanchez- Reyes L. Anthropometric indexes in the prediction of type 2 diabetes mellitus, hypertension and dyslipidaemia in a Mexican population. Int J Obes Relat Metab Disord 2001, 25: $1794-1799$.

35. Kabagambe EK, Baylin A, Campos H. Non-fatal acute myocardial infarction in Costa Rica: Modifiable risk factors, population attributable risk, and adherence to dietary guidelines. Circulation 2007; 115: 1075-1081.

36. Manzur F, Alvear C, Alayón A. Caracterización fenotípica y metabólica del síndrome metabólico en Cartagena de Indias. Rev Colomb Cardiol 2008; 15 97-101.

37. Sánchez F, Jaramillo N, Vanegas A, Echeverría JG, León AC, Echeverrí E, et al. Prevalencia y comportamiento de los factores de riesgo del síndrome metabólico según los diferentes intervalos de edad, en una población femenina del área de influencia de la Clínica Las Américas, en Medellín -Colombia. Rev Colomb Cardiol 2008; 15: 102-110.

38. Villegas A, Botero J, Arango I, Arias S, Toro M. Prevalencia del síndrome metabólico en El Retiro, Antioquia, Colombia. Iatreia 2003; 16: 291-297.

39. Merchán A. Síndrome metabólico y riesgo de enfermedad cardiovascular. Acto Med Colomb 2005; 30: 150-154.

40. Lombo B, Villalobos C, Tique C, Satizábal C, Franco C. Prevalencia de síndrome metabólico entre los pacientes que asisten al servicio de la clínica de hipertensión de la Fundación Santa Fe de Bogotá. Rev Colomb Cardiol 2006; 12: $472-478$.

41. Aschner P. Síndrome metabólico en una población rural y una población urbana de la región andina colombiana. Rev Med 2007; 15: 154-162.

42. Márquez-Sandoval F, Macedo-Ojeda G, Viramontes-Hörner D, FernándezBallart JD, Salas Salvadó J, Vizmanos B. The prevalence of metabolic syndrome in Latin America: a systematic review. Public Health Nutr 2011; 14: $1702-1713$

43. Guerrero-Romero F, Rodríguez M, Sandoval F, Alvarado R. Prevalence of hypertension in indigenous inhabitants of traditional communities from north of Mexico. J Hum Hypertens 2000; 14: 555-559.

44. Sichieri R. Dietary patterns and their association with obesity in the Brazilian city of Rio de Janeiro. Obes Res 2002; 10: 42-48. 
45. Arroyo P, Loria A, Fernandez V, Flegal KM, Kuri P. Prevalence of pre-obesity in urban adult Mexicans in comparison with other large surveys. Obes Res 2000; 8: $179-185$.

46. Sereday M, Gonzalez C, Giorgini P, De Loredo L, Braguinsky J, Cobeñas C, et al. Prevalence of diabetes and obesity in the central area of Argentina. Diabetes Metab 2003; 29: 5S28-5S43.

47. Wilks R, Rotimit C, Bennet F, McFarlane-Anderson N, Kaufmant JS, Anderson SG, et al. Diabetes in the Caribbean: results of a population survey from Spanish Town, Jamaica. Diab Med 1999; 16: 875-883.

48. Piskorz, D. Factores de riesgo en la ciudad de Rosario. Resultados del Estudio FAROS. Revista de la Federación Argentina de Cardiología 1999; 64: 245-251.

49. Avila Curiel A, Shamah- Levy T, Chávez - Villasana A, Galindo Gómez C. Encuesta Urbana de Alimentación y Nutrición en la Zona Metropolitana de la ciudad de México 2002. México DF: Instituto Nacional de Ciencias Médicas y Nutrición Salvador Zubirán, Instituto Nacional de Salud Pública, 2003.

50. De Onis M, Blössnner M. Prevalence and trends of overweight among preschool children in developing countries. Am J Clin Nutr 2000; 72: 1032-1039.

51. Martorell R, Kettel Khan L, Hughes M, Grummer-Strawn. Obesity in Latin American women and children. J Nut 1998; 128: 1464-1473.

52. Monteiro CA. Epidemiologia da Obesidade In: Halpern A, Matos AFG, Suplicy H, Mancini MC \& Zanella MT (eds). Obesidade. Editorial Lemos: São Paulo, 1998; pp.15-30.

53. Pisabarro R, Gutiérrez M, Bermúdez C, Préndez D, Recalde A, Chaftare Y, Manfredi A. Segunda encuesta nacional de sobrepeso y obesidad (ENSO 2) en adultos. Rev Med Urug 2009; 25: 14-26.

54. Orduñez P, Espinosa A, Cooper R, Kaufman J, Nieto F. Hypertension in Cuba: evidenceofnarrowblack-whitedifference. J Hum Hypertens 1998; 12: 111-116.

55. Magalhães N, Pozzan R, Brandão AA, Cerqueira R, Rousoulieres A, Szwarcwald C, Brandão A. Earlybloodpressurelevel as a markof familial aggregationofmetabolic cardiovascular riskfactors. The Rio de Janeiro Study. J Hypertens 1998; 6: 1885-1889.

56. Díaz ME. Hypertensionandobesity. J Hum Hypertens 2002; 16 (Suppl 1): S18-S22.

57. Filozof C, Gonzalez C, Sereday M, Mazza C, Braguinsky J. Obesity prevalence and trends in Latin American countries. Obes Rev 2001; 2: 99-106.

58. Samper-Ternent R, Michaels-Obregon A, Wong R. Coexistence of obesity and anemia in older Mexican adults. Ageing Int 2011; 37: 104-117.

59. Monteiro CA, Moura EC, Conde WL, Popkin BM. Socioeconomic status and obesity in adult populations of developing countries: a review. Bull World Health Organ 2004; 82: 940-946.

60. Monteiro CA, Conde WL, Lu B, Popkin BM. Obesity and inequities in health in the developing world. Int J Obes Relat Metab Disord 2004; 28: 1181-1186.

61. Williams K, Stern MP, Gonzalez-Villalpando C. Secular trends in obesity in Mexico City and in San Antonio. Nutr Rev 2004; 62: S158-S162.

62. Alberti KG, Eckel RH, Grundy SM, Zimmet PZ, Cleeman JI, Donato KA, et al. Harmonizing the metabolic syndrome. A Joint Interim Statement of the International Diabetes Federation Task Force on Epidemiology and Prevention; National Heart, Lung, and Blood Institute; American Heart Association; World Heart Federation; International Atherosclerosis Society; and International Association for the Study of Obesity. Circulation 2009; 120: 1640-1645.

63. Reaven GM. Insulin resistance/compensatory hyperinsulinemia, essential hypertension, and cardiovascular disease. J Clin Endocrinol Metab 2003; 88: 2399-2403.

64. Aschner P, Buendía R, Brajkovich I, Gonzalez A, Figueredo R, Juarez XE, et al. Determination of the cut-off point for waist circumference that establishes the presence of abdominal obesity in Latin American men and women. Diabetes Res Clin Pract 2011; 93: 243-247.

65. American Diabetes Association. Executive summary: standards of medical care in diabetes. 2010. Diabetes Care 2010; 33(Suppl 1): S4-S10.

66. Schriger DL, Lorber B. Lowering the cut point for impaired fasting glucose: where is the evidence? Where is the logic?. Diabetes Care 2004; 27: 592-601.

67. Rutter MK, Nesto RW. Blood pressure, lipids and glucose in type 2 diabetes: how low should we go? Re-discovering personalized care. Eur Heart J 2011; 32: $2247-2255$

68. Jonsson B. Revealing the cost of Type II diabetes in Europe. Diabetologia 2002; 45: S5-S12.

69. Chobanian AV, Bakris GL, Black HR, Cushman WC, Green LA, Izzo JL Jr, et al. The Seventh Report of the Joint National Committee on Prevention, Detection, Evaluation, and Treatment of High Blood Pressure.The JNC 7 report. JAMA 2003; 289: 2560-2572.

70. Mancia G, De Backer G, Dominiczak A, Cifkova R, Fagard R, Germano
G, et al. 2007 ESH-ESC Practice Guidelines for the Management of Arterial Hypertension: ESH-ESC Task Force on the Management of Arterial Hypertension. J Hypertens 2007; 25: 1105-1187.

71. Mancia G, Laurent S, Agabiti-Rosei E, Ambrosioni E, Burnier M, Caufield MJ, et al. Reappraisal of European guidelines on hypertension management: a European Society of Hypertension Task Force document. J Hypertens 2009; 27: 2121-2158.

72. Staessen JA, Asmar R, De Buyzere M, Imai Y, Parati G, Shimada K, et al. Participants of the 2001 Consensus Conference on Ambulatory Blood Pressure Monitoring. Task Force II. Blood pressure measurement and cardiovascular outcome. Blood Press Monit 2001; 6: 355-370.

73. O'Brien E, Asmar R, Beilin L, Imai Y, Mallion JM, Mancia G, et al. European Society of Hypertension Working Group on Blood Pressure Monitoring. European Society of Hypertension recommendations for conventional, ambulatory and home blood pressure measurement. J Hypertens 2003; 21: 821-848.

74. Bonow RO, Mitch WE, Nesto RW, O'Gara PT, Becker RC, Clark LT, et al. Prevention conference VI. Diabetes and Cardiovascular disease. Writing Group V: Management of Cardiovascular- Renal Complications. Circulation 2002; 105: $159-164$

75. Bakris GL, Williams M, Dworkin L, Elliott WJ, Epstein M, Toto R, et al. Preserving renal function in adults with hypertension and diabetes: a consensus approach. Am J Kidney Dis 2000; 36: 646-661.

76. DeFronzo RA. Diabetic nephropathy: etiologic and therapeutic considerations. Diabetes Rev 1995; 3: 510-564.

77. Malmberg K, Norhammar A, Wedel H, Ryden L. Glycometabolic state at admission: important risk marker of mortality in conventionally treated patients with diabetes mellitus and acute myocardial infarction: long term results from the Diabetes and Insulin-glucose Infusion in Acute Myocardial Infarction (DIGAMI study). Circulation 1999; 99: 2626-2632.

78. Colwell JA. Aspirin Therapy in diabetes (Technical Review). Diabetes Care 1997; 20: 1767-1771.

79. McDonagh TA, Morrison CE, Lawrence A, Ford I, Tunstall-Pedoe H, McMurray JJ, Dargie HJ. Symptomatic and asymptomatic left ventricular systolic dysfunction in an urban population. Lancet 1997; 350: 829-833.

80. Iribarren C, Karter AJ, Go AS, Ferrara A, Liu JY, Sidney S, Selby JV. Glycaemic control and HF among adult patients with diabetes. Circulation 2001; 103: 2668-2673.

81. Bell DS. Heart Failure: the frequent, forgotten and often fatal complication of diabetes. Diabetes Care 2003; 26: 2433-2441.

82. Marantz PR, Tobin JN, Wassertheil-Smoller S, Steingart RM, Wexler JP Budner N, et al. The relationship between left ventricular function and congestive HF diagnosed by clinical criteria. Circulation 1988; 77: 607-612.

83. Kasrapanayiotides T, Piechowski-Jozwiak B, Van Melle G, Bogousslavsky J, Devuyst G. Stroke Patterns, etiology and prognosis in patients with diabetes mellitus. Neurology 2004; 62: 1558-1562.

84. Megherbi SE, Milan C, Minier D, Couvreur G. Osseby GV, Tilling K, et al. Association between diabetes and stroke subtype on survival and functional outcome 3 months after stroke: data from the European BIOMED Stroke Project. Stroke 2003; 34: 688-694.

85. Stevens RJ, Coleman RL, Adler AI, Stratton IM, Matthews DR, Holman RR. Risk factors for myocardial infarction case fatality and stroke case fatality in type 2 diabetes: UKPDS 66. Diabetes Care 2004; 27: 201-207.

86. Shepard R, Balady G. Exercise as cardiovascular therapy. Circulation 1999; 99: $963-972$

87. American Diabetes Association. Position Statement. Diabetesmellitus and exercise. Diabetes Care 2000; 23(suppl 1): S50-S54.

88. Walker KZ, Piers LS, Putt RS Jones JA, O’ Dea K. Effects of regular walking on cardiovascular risk factors and body composition in normoglycaemic woman and woman with type 2 diabetes. Diabetes Care 1999; 22: 555-561.

89. Hansson L, Zanchetti A, Carruthers SG, Dahlof B, Elmfeldt D, Julius S, et al.Effects of intensive blood-pressure lowering and low-dose aspirin in patients with hypertension: principal results of the Hypertension Optimal Treatment (HOT) randomised trial. Lancet 1998; 351: 1755-1762.

90. UK Prospective Diabetes Study Group. Tight blood pressure control and risk of macrovascular and microvascular complications in type 2 diabetes: UKPDS 38. BMJ 1998; 317: 703-713.

91. Curb JD, Pressel SL, Cutler JA, Savage PJ, Applegate WB, Black H, et al. Effect of diuretic-based antihypertensive treatment on cardiovascular disease risk in older diabetic patients with isolated systolic hypertension. Systolic Hypertension in the Elderly Program Cooperative Research Group. JAMA 1996; 276: $1886-1892$

92. Tuomilehto J, Rastenyte D, Birkenhäger WH, Thijs L, Antikainen R, Bulpitt CJ, et al. Effects of calcium-channel blockade in older patients with diabetes 
and systolic hypertension. Systolic Hypertension in Europe Trial Investigators. N Engl J Med 1999; 340: 677-684.

93. Estacio RO, Jeffers BW, Gifford N, Schrier RW. Effect of blood pressure control on diabetic microvascular complications in patients with hypertension and type 2 diabetes. Diabetes Care 2000; 23(Suppl 2): S54-S64.

94. Heart Outcomes Prevention Evaluation (HOPE) Study investigators. Effects of ramipril on cardiovascular and microvascular outcomes in people with diabetes mellitus: results of the HOPE study and MICROHOPE substudy. Lancet 2000; 355: $253-259$.

95. Cooper-DeHoff RM, Gong Y, Handberg EM, Bavry AA, Denardo SJ, Bakris GL, Pepine CJ. Tight blood pressure control and cardiovascular outcomes among hypertensive patients with diabetes and coronary artery disease. JAMA 2010; 304: 61-68.

96. Zanchetti A, Grassi G, Mancia G. When should antihypertensive drug treatment be initiated and to what levels should systolic blood pressure be lowered? A critical reappraisal. J Hypertens 2009; 27: 923-934.

97. ACCORD Study group. Effects of intensive blood-pressure control in type 2 diabetes mellitus. $N$ Engl J Med 2010; 362: 1575-1585.

98. Bangalore S, Kumar S, Lobach I, Messerli FH. Blood pressure targets in subjects with type 2 diabetes mellitus/impaired fasting glucose: observation from traditional and Bayesian random-effects meta-analysis of randomized trials. Circulation 2011; 123: 2799-2810.

99. Reboldi G, Gentile G, Angeli F, Ambrosio G, Mancia G, Verdecchia P. Effects of intensive blood pressure reduction on myocardial infarction and stroke in diabetes: a meta-analysis in 73,913 patients. J Hypertens 2011; 29: 1253 -1169 .

100. Patel A. ADVANCE Collaborative Group, MacMahon S, Chalmers J, Neal B, Woodward M, et al. Effects of a fixed combination of perindropil and indapamide on macrovascular and microvascular outcomes in patients with type 2 diabetes mellitus (the ADVANCE trial). Lancet 2007; 370: 829-840.

101. Lewis JB. Blood pressure control in chronic kidney disease: is less really more? J Am Soc Nephrol 2010; 21: 1086-1092.

102. Turnbull F, Neal B, Ninomiya T, Algert C, Arima H, et al. Blood Pressure Lowering Treatment Trialists' Collaboration. Effects of different blood pressure-lowering regimens on major cardiovascular events in individuals with and without diabetes mellitus: results of prospectively designed overviews of randomized trials. Arch Intern Med 2005; 165: 1410-1419.

103. Yusuf S, Teo KK, Pogue J, Dyal L, Copland I, et al. ONTARGET Investigators. Telmisartan, ramipril, or both in patients at high risk for vascular events. $N$ Engl J Med 2008; 358: 1547-1559.

104. Jamerson K, Weber MA, Bakris GL, Dahlöf B, Pitt B, Shi V, et al. Benazepril plus amlodipine or hydrochlorothiazide for hypertension in high-risk patients. N Engl J Med 2008; 359: 2417-2428.

105. U.S. Food and Drug Administration. FDA Drug Safety Communication: New Warning and Contraindication for blood pressure medicines containing aliskiren (Tekturna). Rockville, USA: U.S. Food and Drug Administration; 2012. (cited 2012 Sep 10). Available from: http://www.fda.gov/drugs/drugsafety/ucm300889. htm

106. Stears AJ, Woods SH, Watts M, Burton TJ, Graggaber J, Mir FA, Brown MJ. A double-blind, placebo-controlled, crossover trial comparing the effects of amiloride and hydrochlorothiazide on glucose tolerance in patients with essential hypertension. Hypertension 2012; 59: 934-942.

107. Central Intelligence Agency-CIA. The World Factbook. Washington, USA: Central Intelligence Agency-CIA; 2012. (cited 2012 Aug 10). Available from: https://www.cia.gov/library/publications/the-world-factbook/wfbExt/ region_soa.html

108. Sanchez-Johnsen LA, Fitzgibbon ML, Martinovich Z, Stolley MR, Dyer AR, Van Horn L. Ethnic differences in correlates of obesity between Latin-American and black women. Obes Res 2004; 12: 652-660.

109. Barcelo A, Gregg EW, Partor-Valero M, Robles SC. Waist circumference, BMI and the prevalence of self-reported diabetes among the elderly of the United States and six cities of Latin American and the Caribbean. Diabetes Res Clin Pract 2007; 78: 418-427.

110. Anselmi M, Avanzini F, Moreira JM, Montalvo G, Armani D, Prandi R, et al. Treatment and control of arterial hypertension in a rural community in Ecuador. Lancet 2003; 36: 1186-1187.

111. Flack JM, Sica DA, Bakris G, Brown AL, Ferdinand KC, Grimm Jr RH, et al. Management of High Blood Pressure in Blacks. An Update of the International Society on Hypertension in Blacks Consensus Statement on behalf of the International Society on Hypertension in Blacks. Hypertension 2010, 56: 780-800.

112. Burke GL, Savage PJ, Manolio TA, Sprafka JM, Wagenknecht LE, Sidney S, et al. Correlates of obesity in young black and white women: the CARDIA
Study. Am J Public Health 1992; 82: 1621-1625.

113. Sharp TA, Bell ML, Grunwald GK, Schmitz KH, Sidney S, Lewis CE, et al. Differences in resting metabolic rate between white and African-American young adults. Obes Res 2002; 10: $726-732$.

114. McVlellan W, Tuttle E, Issa A. Racial Differences in the incidence of hypertensive end-stage renal disease. Am J Kidney Dis 1998; 12: 285-290

115. Lopes AA, Port FK. The low birth weight hypothesis as a plausible explanation for the black/white differences in hypertension, non-insulin-dependent diabetes, and end-stage renal disease. Am J Kidney Dis 1995; 25: 350.

116. Roger VL, Go AS, Lloyd-Jones DM, Adams RJ, Berry JD, Brown TM, et al. Heart Diseases and stroke statistics: 2011 update a report from de American Heart Association. Circulation 2011; 123: 18-1209.

117. Sareli P, Radevski IV, Valtchanova ZP, Libhaber E, Candy GP, Den Hond E, et al. Efficacy of different drug classes used to initiate antihypertensive treatmen in black subjects: results of a randomized trial in Johannesburg, South Africa. Arch Intern Med 2001; 161(7): 965-971.

118. Wright JT Jr, Dunn JK, Cutler JA, Davis BR, Cushman WC, Ford CE, et al. Outcomes in hypertensive black and nonblack patients treated with chlorthalidone, amlodipine, and lisinopril. JAMA 2005; 293(13): 1595-608.

119. Brown NJ, Ray WA, Snowden M, Griffin MR. Black Americans have an in creased rate of angiotensin converting enzyme inhibitor-associated angioedema. Clin Pharmacol Ther 1996; 60(1): 8-13.

120. Medina-Lezama J, Zea-Diaz H, Morey-Vargas OL, Bolaños-Salazar JF, Postigo-Macdowall M, Paredes-Diaz S, et al. Prevalence and patterns of hypertension in Peruvian Andean Hispanics: the PREVENCION study. $J$ Am SocHypertens 2007; 1: 216-225.

121. Agusti R. Epidemiologia de la hipertensión arterial en el Perú. Acta Med Per 2006; 23(2): 69-75.

122. Organización Panamericana de la Salud. Base de Datos de Indicadores Básicos en Salud de la OPS. Washington: OPS; 2007. (cited 2012 Sep 10). Available from: http://www.paho.org/spanish/dd/ais/cp_152.htm

123. Vasan RS, Beiser A, Seshadri S, Larson MG, Kannel WB, D'Agostino RB, Levy D. Residual lifetime risk for developing hypertension in middle-aged women and men: the Framingham Heart Study. JAMA 2002; 287: 1003-1010.

124. Benetos A, Safar M, Rudnichi A, Smulyan H, Richard JL, Ducimetiére $\mathbf{P}$, Guize J. Pulse pressure: a predictor of long-term cardiovascular mortality in a French male population. Hypertension 1997; 30: 1410-1415.

125. Silagy CA, McNeil JJ, Farish S, McGrath BP. Comparation of repeated measurement of ambulatory and clinic blood pressure readings in isolated systolic hypertension. ClinExp Hypertension 1995; 15: 895-909.

126. Wiinberg N, Hoegholm A, Christensen HR, Bang LE, Mikkelsen KL, Nielsen PE, et al. 24-h-ambulatory blood pressure in 352 normal Danish subjects, related to age and gender. Am J Hypertens 1995; 8: 978-986.

127. Kario K, Pickering TG, Matsuo T, Hoshide S, Schwartz JE, Shimada K. Stroke prognosis and abnormal nocturnal blood pressure falls in older hypertensive. Hypertension 2001; 38: 852-857.

128. Kario K, Pickering TG, Umeda Y, Hoshide S, Hoshide Y, Morinari M, et al. Morning surge in blood pressure as a predictor of silent and clinical cerebrovascular disease in elderly hypertensives: a prospective study. Circulation 2003; 107: 1401-1406

129. Verdecchia P, Angeli F, Mazzotta G, Garofoli M, Ramundo E, Gentile G, et al. Day-night dip and early-morning surge in blood pressure in hypertension: prognostic implications. Hypertension 2012; 60: 34-42.

130. Beckett NS, Peters R, Fletcher AE, Staessen JA, Liu L, Dumitrascu D, et al. HYVET Study Group, Treatment of hypertension in patients 80 years of age or older. N Eng J Med 2008; 358: 1887-1898.

131. SHEP Cooperative Research Group. Prevention of stroke by antihypertensive drug treatment in older persons with isolated systolic hypertension.Final result of the Systolic Hypertension in the Elderly Program (SHEP). JAMA 1991; 265(24): 3255-3264

132. Staessen JA, Fagard R, Thijs L, Celis H, Arabidze GG, Birkenhäger WH, et al. Randomised double-blind comparison of placebo and active treatment for older patients with isolated systolic hypertension. The Systolic Hypertension in Europe (Syst-Eur) Trial Investigators. Lancet 1997; 350: 757-764.

133. Liu L, Wang JG, Gong L, Liu G, Staessen JA. comparison of active treatmen and placebo in older Chinese patients with isolated systolic hypertension, Systolic Hypertension in China (SYST-CHINA) Collaborative Group. J Hypertension 1998; 16: 1823-1829.

134. Lopez-Jaramillo P, Lahera V, Lopez-Lopez J. Epidemic of cardiometabolic diseases: A Latin American point of view. Ther Adv Cardiovasc Dis 2011; 5: 119-131.

135. López-Jaramillo P, Silva SY, Rodríguez Salamanca N, Duran A, Mosquera W, Castillo V. Are Nutrition-Induced Epigenetic Changes the Link Between 
Socioeconomic Pathology and Cardiovascular Diseases? Am J Therap 2008; 15: $362-372$.

136. López-Jaramillo P. Cardio-metabolic disease in Latin America: the role of fetal programming in response to maternal malnutrition. Rev Esp Cardiol 2009; 62: 670-676.

137. Fernandes MT, Sesso R, Martins PA, Sawaya AL. Increased blood pressure in adolescents of socioeconomic status with short stature. Pediatr Nephrol 2003; 18: $435-439$.

138. Florêncio TT, Ferreira HS, Cavalcante JC, Sawaya AL. Short stature, obesity and arterial hypertension in a very low income population in North-eastern Brazil. Nutr Metab Cardiovasc Dis 2004; 14: 26-33.

139. Ferreira HS, Moura FA, Cabral CR Jr, Florêncio TM, Vieira RC, de Assunção ML. Short stature of mothers from an area endemic for under-nutrition is associated with obesity, hypertension and stunted children: A population-based study in the semi-arid region of Alagoas, Northeast Brazil. Br J Nutr 2009; 101: $1239-1245$.
140. López-Jaramillo P, Herrera E, Garcia RG, Camacho PA, Castillo VR. Interrelationships between body mass index, $\mathrm{C}$-reactive protein and blood pressure in a Hispanic pediatric population. Am J Hypertens 2008; 21: 527-532.

141. Franco MC, Casarini DE, Carneiro-Ramos MS, Sawaya AL, BarretoChaves ML, Sesso R. Circulating renin-angiotensin system and catecholamines in childhood: is there a role for birth weight? Clin Sci (Lond) 2008: 114: 375-380.

142. Febba A, Sesso R, Barreto GP, Liboni CS, Franco MC, Casarini DE. Stunting growth: Association of the blood pressure levels and ACE activity in early childhood. Pediatr Nephrol 2009; 24: 379-386.

143. González-Barranco J, Ríos-Torres JM, Castillo-Martínez L, López-Alvarenga JC, Aguilar-Salinas CA, Bouchard C, et al. Effect of malnutrition during the first year of life on adult plasma insulin and glucose tolerance. Metabolism 2003; 52: 1005-1011.

144. Lopez-Jaramillo P. Defining the research priorities to fight the burden of cardiovascular diseases in Latin America. J Hypertens 2008; 26: 1886-1889. 